\title{
Econometric Estimation in Long-Range Dependent Volatility Models: Theory and Practice
}

\author{
IsABel CASAS ${ }^{1,2}$ AND Jiti GaO ${ }^{2}$ \\ ${ }^{1}$ Department of Statistics and Econometrics, Universidad Carlos III de Madrid, C/Madrid, \\ 126, 28903-Getafe (Madrid) Spain. \\ ${ }^{2}$ School of Mathematics and Statistics, The University of Western Australia, Crawley WA \\ 6009, Australia.
}

\begin{abstract}
It is commonly accepted that some financial data may exhibit long-range dependence, while other financial data exhibit intermediate-range dependence or short-range dependence. These behaviours may be fitted to a continuous-time fractional stochastic model. The estimation procedure proposed in this paper is based on a continuous-time version of the Gauss-Whittle objective function to find the parameter estimates that minimize the discrepancy between the spectral density and the data periodogram. As a special case, the proposed estimation procedure is applied to a class of fractional stochastic volatility models to estimate the drift, standard deviation and memory parameters of the volatility process under consideration. As an aplication, the volatility of the Dow Jones, S\&P 500, CAC 40, DAX 30, FTSE 100 and NIKKEI 225 is estimated.
\end{abstract}

KEYWORDS: Continuous-time model, diffusion process, long-range dependence, stochastic volatility.

JEL Subject Classification: C13; G13 


\section{INTRODUCTION}

There is a long history about the study of stochastic volatilities. More than thirty years ago, Black and Scholes (1973) assume a constant volatility to derive their famous option pricing equation. The implied volatility values obtained from this equation show skewness, suggesting that the assumption of constant volatility is not feasible. In fact, the volatility shows an intermittent behaviour with periods of high values and periods of low values. In addition, the asset volatility cannot be directly observed. Stochastic volatility (SV) models deal with these two facts. Taylor (1986) and Hull and White (1987) were amongst the first to study the logarithm of the stochastic volatility as an OrnsteinUhlenbeck process. A review and comparative study about modeling SV up to 1994 has been given by Taylor (1994). Andersen and Sørensen (1996) examine generalized moments of method for estimating stochastic volatility model. Andersen and Lund (1997) extend the CIR model proposed by Cox, Ingersoll and Ross (1985) to associate the spot interest rate with stochastic volatility process through estimating the parameters with the efficient method of moments. The main assumption of the SV model is that the volatility is a log-normal process. The probabilistic and statistical properties of a lognormal are well known. However, parameter estimation has not been uncomplicated due to the difficulty finding the maximum likelihood (ML) function. The authors also like to mention the nonuniqueness of the log-normal distribution as reviewed by Stoyanov (2004). In addition to the log-normal distribution, Barndorff-Nielsen and Leonenko (2005) study other possible distributions of the volatility process, such as the Gamma and inverse Gaussian distributions. A recent review about the development of multivariate stochastic volatility is given in Asai, McAleer and Yu (2006).

Recent studies show that some data sets may display long-range dependence (LRD). See, for example, the recent surveys by Beran 1994, Baillie and King 1996, Anh and Heyde 1999, and Robinson 2003. Since about ten years ago, there has been some work on studying stochastic volatility with LRD. Breidt, Crato and De Lima (1998), Comte and Renault (1998), and Harvey (1998) were among the first to consider long-memory stochastic volatility (LMSV) models. Meanwhile, Breidt, Crato and De Lima (1998), and Harvey (1998) independently consider a LMSV case where the log-volatility is modelled as a fractionally integrated ARMA (ARFIMA) process. Comte and Renault (1998) consider a continuous-time fractionally stochastic volatility (FSV) model of the form

$$
d Y(t)=v(t) d B(t) \quad \text { and } \quad d x(t)=-\alpha x(t) d t+\sigma d B_{\beta}(t)
$$

where $x(t)=\ln (v(t)), Y(t)=\ln (S(t))$ with $S(t)$ being the return process, $B(t)$ is a 
standard Brownian motion, $\alpha$ is the drift parameter involved in the stochastic volatility process, $\sigma>0$ is the volatility parameter involved in the stochastic process, and $B_{\beta}(t)$ is a fractionally Brownian motion process of the form: $B_{\beta}(t)=\int_{0}^{t} \frac{(t-s)^{\beta}}{\Gamma(1+\beta)} d B_{1}(s)$, where $B_{1}(t)$ is another standard Brownian motion and $\Gamma(x)$ is the usual $\Gamma$ function. A process displays LRD if $0<\beta<\frac{1}{2}$. When $\beta=0$, the process is called short-range dependent. The process is called intermediate-range dependent if $-\frac{1}{2}<\beta<0$ (see $\S 12.4$ of Brockwell and Davis 1990 for more precise definitions). Comte and Renault (1998) propose a discretization procedure to approximate the solution of their continuous-time FSV model. An estimation procedure for $0<\beta<\frac{1}{2}$ is then developed for a discretized version of the solution $Y(t)$ based on the so-called log-periodogram regression. Recently, Deo and Hurvich (2001) also study such an estimation procedure based on the logperiodogram regression method, and then establish the mean-squared error properties as well as consistency results for an estimator of $\beta$ using the so-called GPH estimator initially proposed by Geweke and Porter-Hudak (1983). Gao (2004) points out that it is possible to estimate all the parameters involved in model (1) using the so-called continuous-time version of the Gauss-Whittle contrast function method proposed in Gao, et al. (2001) and Gao, Anh and Heyde (2002). Other closely related papers include Giraitis and Surgailis (1990), Hosoya (1997), Sun and Phillips (2003), Andrews and Sun (2004), Anh, Leonenko and Sakhno (2004), and Leonenko and Sakhno (2006).

This paper considers a class of stochastic volatility models of the form

$$
d Y(t)=V(t) d B(t)
$$

with $V(t)$ being given by $V(t)=e^{X(t)}$, in which

$$
X(t)=\int_{-\infty}^{t} A(t-s) d B_{1}(s)
$$

where $A(\cdot)$ is a deterministic function such that $X(t)$ is stationary, and the explicit expression of $A(\cdot)$ is determined by the spectral density of $X(t)$. Let $\gamma(\tau)=E[X(t) X(t+$ $\tau)$ ]. In addition, we assume that the spectral density function of $X(t)$ is defined by

$$
\phi_{X}(\omega)=\phi_{X}(\omega, \theta)=\frac{1}{2 \pi} \int_{-\infty}^{\infty} e^{-i \tau \omega} \gamma(\tau) d \tau=\frac{\psi(\omega, \theta) \sigma^{2}}{|\omega|^{2 \beta}\left(\omega^{2}+\alpha^{2}\right)}, \quad \omega \in(-\infty, \infty),
$$

where $\theta=(\alpha, \beta, \sigma) \in \Theta=\left\{0<\alpha<\infty,-\frac{1}{2}<\beta<\frac{1}{2}, 0<\sigma<\infty\right\}, \psi(\omega, \theta)$ is a either a parametric function of $\theta$ or a semiparametric function of $\theta$ and an unknown, continuous

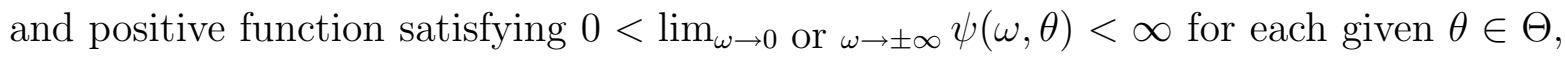
$\alpha$ is normally involved in the drift function of the stochastic volatility process, $\beta$ is 
the memory parameter, and $\sigma$ is a kind of volatility of the stochastic volatility process. In this paper, we also assume that $B(s)$ and $B_{1}(t)$ are mutually independent for all $-\infty<s, t<\infty$. The case where $B(s)$ and $B_{1}(t)$ may be dependent is mentioned below Assumptions 2.1 and 2.2 .

Unlike most existing studies assuming a particular form for the volatility process $V(t)$, we only implicitly impose certain conditions on the distributional structure of the volatility process. First, the volatility is a log-normal process with a vector of parameters involved. Second, the vector of parameters is specified through a corresponding spectral density function. Third, the parameters involved in the spectral density function may be explicitly interpreted and fully estimated. Fourth, the generality of the spectral density function of the form (4) implicitly implies that the class of log-normal volatility processes can be quite general. As a matter of the fact, the class of models (2)-(4) is quite general to cover some existing models. For example, when $\psi(\omega, \theta)=\frac{1}{\Gamma^{2}(1+\beta)}$, model (4) reduces to

$$
\phi_{X}(\omega)=\phi_{X}(\omega, \theta)=\frac{\sigma^{2}}{\Gamma^{2}(1+\beta)} \frac{1}{|\omega|^{2 \beta}} \frac{1}{\omega^{2}+\alpha^{2}},
$$

which is just the spectral density of the solutions of the second equation of (1) given by

$$
x(t)=\int_{0}^{t} A(t-s) d B_{1}(t) \text { with } A(x)=\frac{\sigma}{\Gamma(1+\beta)}\left(x^{\beta}-\alpha \int_{0}^{x} e^{-\alpha(x-u)} u^{\beta} d u\right) .
$$

Its stationary version is defined as $X(t)=\int_{-\infty}^{t} A(t-s) d B_{1}(t)$. Existence of some other models corresponding to (3) has been established by Comte and Renault (1996), Anh, Heyde and Leonenko (2002), Anh and Inoue (2005), and Anh, Inoue and Kasahara (2005). As discussed in the these papers, models with solutions given by (3) and having a spectral density of the form (4) include a mean-reverting model with a fractional noise of the form

$$
d X(t)=a(b-X(t)) d t+c d B_{2}(t),
$$

where $a, b$ and $c$ are probably unknown parameters and $B_{2}(t)$ or its stationary version is driven by $B_{2}(t)=\int_{-\infty}^{t} C(t-s) d B_{3}(t)$, in which $B_{3}(t)$ is also a standard Brownian motion and $C(\cdot)$ is a deterministic function. Model $(7)$ is indeed the natural extension of the seminal model by Vasicek (1977) to a long-range dependent setting.

Instead of further discussing such existence, we concentrate on the parameter estimation of the stochastic volatility process (3) through estimating the parameters involved in the spectral density function (4). We then demonstrate how to implement such an estimation procedure in practice through using both simulated and real sets of data.

The main contribution of this paper can be summarized as follows: (i) it considers a general class of stochastic volatility models with either long-range dependence (LRD), 
intermediate-range dependence (IRD), or short-range dependence (SRD); (ii) it proposes an estimation procedure to deal with cases where a class of non-Gaussian processes may display LRD, IRD or SRD; (iii) our comprehensive simulation studies show that the proposed estimation procedure works well numerically not only for the memory parameter $\beta$, but also for both the drift parameter $\alpha$ and the variance $\sigma^{2}$; and (iv) the methodology is also applied to the estimation of the volatility of several well-known stock market indexes.

This paper is organised as follows. Section 2 proposes an estimation procedure for non-Gaussian processes with possible LRD, IRD and SRD. Asymptotic properties for such an estimation procedure are established in Section 2. The numerical implementation of the proposed estimation procedure is given in Section 3. Section 4 gives some application of the proposed estimation procedure to several well-known stock market indexes. These results are compared with those based on the GPH estimation. Section 5 concludes the paper with some remarks. Mathematical details are relegated to Appendix A. Appendix B contains all the tables and figures.

\section{Estimation Procedure}

Since observations on $Y(t)$ are made at discrete intervals of time in many practical circumstances, even though the underlying process may be continuous, we propose an estimation procedure based on a simple first-order approximate version of $Y(t)$ in $(2)$. It should be mentioned that other types of approximations may also be possible. We also like to point out that using a higher-order approximate version may not be optimal in terms of minimizing approximate biases as studied in Fan and Zhang (2003).

Consider a discretized version of model (2) of the form

$$
Y_{t \Delta}-Y_{(t-1) \Delta}=V_{(t-1) \Delta}\left(B_{t \Delta}-B_{(t-1) \Delta}\right), t=1,2, \cdots, T
$$

where $\Delta$ is the time between successive observations and $T$ is the size of observations. In theory, we may study asymptotic properties for our estimation procedure for either the case where $\Delta$ is small but fixed or the case where $\Delta$ is varied according to $T$ (see Arapis and Gao 2006). We focus on the case where $\Delta$ is small but fixed throughout the rest of this paper, since this paper is mainly interested in estimating stochastic volatility process $V(t)$ using either monthly, weekly, daily or higher frequency returns.

Let $W_{t}=\frac{Y_{t \Delta}-Y_{(t-1) \Delta}}{\Delta}, U_{t}=V_{(t-1) \Delta}$ and $\epsilon_{t}=\sqrt{\Delta}\left(B_{t \Delta}-B_{(t-1) \Delta}\right)$. Then model (8) may be re-written as

$$
W_{t}=U_{t} \sqrt{\Delta^{-1}} \epsilon_{t}, t=1,2, \cdots, T \text {, }
$$


where $\left\{\epsilon_{t}\right\}$ is a sequence of independent and identically distributed (i.i.d.) normal errors drawn from $N(0,1)$. Letting $Z_{t}=\log \left(W_{t}^{2}\right), X_{t}=\log \left(U_{t}\right), e_{t}=\log \left(\epsilon_{t}^{2}\right)-E\left[\log \left(\epsilon_{t}^{2}\right)\right]$ and $\mu=E\left[\log \left(\epsilon_{t}^{2}\right)\right]-\log (\Delta)$, model (9) implies that

$$
Z_{t}=\mu+2 X_{t}+e_{t}, t=1,2, \cdots, T
$$

where $\left\{X_{t}\right\}$ is a sequence of stationary Gaussian time series with $\operatorname{LRD}$, and $\left\{e_{t}\right\}$ is a sequence of i.i.d. random errors with $E\left[e_{t}\right]=0$ and $\sigma_{e}^{2}=E\left[e_{t}^{2}\right]=\frac{\pi^{2}}{2}$.

Such a simple linear model has been shown to be pivotal for establishing various consistent estimation procedures (Deo and Hurvich 2001). Our estimation procedure is also based on model (10).

Since both $Z_{t}$ and $X_{t}$ are stationary, their corresponding spectral density functions $f_{Z}(\cdot, \cdot)$ and $f_{X}(\cdot, \cdot)$ satisfy the following relationship:

$$
f_{Z}(\omega, \theta)=4 f_{X}(\omega, \theta)+\frac{\sigma_{e}^{2}}{2 \pi}=4 f_{X}(\omega, \theta)+\frac{\pi}{4} .
$$

As $\left\{X_{t}\right\}$ is a sequence of discrete observations of the continuous-time process $\{X(t)\}$, existing results (Priestley 1981, §7.1.1) show that the spectral density function $f_{X}(\omega, \theta)$ of the discrete process for $-\frac{\pi}{\Delta} \leq \omega \leq \frac{\pi}{\Delta}$ is the superposition of the spectral density of the continuous process $\phi_{X}(\omega, \theta)$ for frequencies $\omega, \omega \pm \frac{2 k \pi}{\Delta}, \omega \pm \frac{4 k \pi}{\Delta}, \ldots$ This is expressed as

$$
f_{X}(\omega, \theta)=\sum_{k=-\infty}^{\infty} \phi_{X}\left(\omega+\frac{2 k \pi}{\Delta}, \theta\right)
$$

which, together with (11), implies that the spectral density function of $Z_{t}$ is given by

$$
f(\omega, \theta) \equiv f_{Z}(\omega, \theta)=4 \sum_{k=-\infty}^{\infty} \phi_{X}\left(\omega+\frac{2 k \pi}{\Delta}, \theta\right)+\frac{\pi}{4} \quad-\frac{\pi}{\Delta} \leq \omega \leq \frac{\pi}{\Delta} .
$$

In practice, we can approximate $f_{X}(\omega, \theta)$ arbitrarily well by

$$
\widehat{f}_{M}(\omega, \theta)=\sum_{k=-M}^{M} \phi_{X}(\omega-2 k \pi, \theta),
$$

where $M=M(T)$ is a sufficiently large integer. We can easily show that as $M \rightarrow \infty$, $\widehat{f}_{M}(\omega, \theta)$ converges to $f_{X}(\omega, \theta)$ uniformly over $\omega \neq 0$ and $\theta$.

The spectral density $f(\omega, \theta)$ of (13) can be estimated by the following periodogram

$$
I_{T}(\omega)=I_{T}^{Z}(\omega)=\frac{1}{2 \pi T}\left|\sum_{t=1}^{T} e^{-i \omega t} Z_{t}\right|^{2} .
$$


This paper proposes using a Whittle estimation procedure. Since the exact maximum likelihood estimation (MLE) procedure for short-range dependent Gaussian time series poses computational problems, Whittle (1951) proposes a so-called Whittle estimation method to approximate the exact MLE for parameter estimation of short-range dependent Gaussian time series. The Whittle estimation method has since been extensively studied in the literature for both Gaussian and non-Gaussian cases. See Robinson (2003) for a recent review.

The Whittle contrast function used in this paper is as follows:

$$
W_{T}(\theta)=\frac{1}{4 \pi} \int_{-\pi}^{\pi}\left\{\log (f(\omega, \theta))+\frac{I_{T}(\omega)}{f(\omega, \theta)}\right\} d \omega .
$$

We thus estimate $\theta$ by

$$
\tilde{\theta}_{T}=\arg \min _{\theta \in \Theta_{0}} W_{T}(\theta),
$$

where $\Theta_{0}$ is a compact subset of the parameter space $\Theta$.

As the minimization problem (17) involves the continuous-time version $W_{T}(\theta)$, in practice we propose using an evolutionary algorithm to find the global minimizer $\tilde{\theta}_{T}$ in Sections 3 and 4 below based on a discretized version of $W_{T}(\theta)$ of the form

$$
\bar{W}_{T}(\theta)=\frac{1}{2 T} \sum_{s=1}^{T-1}\left\{\log \left(f\left(\omega_{s}, \theta\right)\right)+\frac{I_{T}\left(\omega_{s}\right)}{f\left(\omega_{s}, \theta\right)}\right\},
$$

where $\omega_{s}=\frac{2 \pi s}{T}$. The resulting estimate $\bar{\theta}_{T}=(\bar{\alpha}, \bar{\beta}, \bar{\sigma})^{\top}$ has the same asymptotic behavior as $\tilde{\theta}_{T}$, because Lemma A.2 in Appendix A remains true when $W_{T}(\theta)$ is replaced by $\bar{W}_{T}(\theta)$.

Equations (15)-(18) have been working well both in theory and practice for the case where the underlying process $\left\{Z_{t}\right\}$ is Gaussian. See, for example, Gao, et al. (2001), Gao, Anh and Heyde (2002), and Gao (2004). Our theory and simulation results below show that such an estimation also works well both theoretically and practically for the case where $\left\{Z_{t}\right\}$ is stationary but non-Gaussian.

To state the main theoretical results of this paper, we need to introduce the following assumptions. For simplicity, we denote $\theta=(\alpha, \beta, \sigma)^{\top}=\left(\theta_{1}, \theta_{2}, \theta_{3}\right)^{\top}$.

Assumption 2.1. (i) Consider the general model structure given by (2)-(4). Suppose that the two standard Brownian motion processes $B(s)$ and $B_{1}(t)$ are mutually independent for all $-\infty<s, t<\infty$.

(ii) Assume that $\psi(\omega, \theta)$ is a positive and continuous function in both $\omega$ and $\theta$, bounded away from zero and chosen to satisfy

$$
\int_{-\pi}^{\pi} f_{X}(\omega, \theta) d \omega<\infty \text { and } \int_{-\pi}^{\pi} \log \left(f_{X}(\omega, \theta)\right) d \omega>-\infty \text { for } \theta \in \Theta
$$


In addition, $\psi(\omega, \theta)$ is a symmetric function in $\omega$ satisfying $0<\lim _{\omega \rightarrow 0} \psi\left(\omega, \theta^{*}\right)<\infty$ and $0<\lim _{\omega \rightarrow \pm \infty} \psi\left(\omega, \theta^{*}\right)<\infty$ for each given $\theta^{*} \in \Theta$.

(iii) Assume that $K\left(\theta, \theta_{0}\right)$ is convex in $\theta$ on an open set $\mathcal{C}\left(\theta_{0}\right)$ containing $\theta_{0}$, where $\theta_{0} \in \Theta_{0}$ is the true value of $\theta$ and

$$
K\left(\theta, \theta_{0}\right)=\frac{1}{4 \pi} \int_{-\pi}^{\pi}\left\{\frac{f\left(\omega, \theta_{0}\right)}{f(\omega, \theta)}-1-\log \left(\frac{f\left(\omega, \theta_{0}\right)}{f(\omega, \theta)}\right)\right\} d \omega .
$$

Assumption 2.2. The functions $f_{X}(\omega, \theta)$ and $g_{i X}(\omega, \theta)=-\frac{\partial f_{X}^{-1}(\omega, \theta)}{\partial \theta_{i}}$ for $1 \leq i \leq 3$ satisfy the following properties:

(i) $\frac{\partial^{2} \int_{-\pi}^{\pi} \log \left(f_{X}(\omega, \theta)\right) d \omega}{\partial^{2} \theta}$ does exists and equals $\int_{-\pi}^{\pi} \frac{\partial^{2} \log \left(f_{X}(\omega, \theta)\right)}{\partial^{2} \theta} d \omega$;

(ii) $f_{X}(\omega, \theta)$ is continuous at all $\omega \neq 0$ and $\theta \in \Theta, f_{X}^{-1}(\omega, \theta)$ is continuous at all $(\omega, \theta)$;

(iii) the inverse function $f_{X}^{-1}(\omega, \theta), \omega \in(-\pi, \pi], \theta \in \Theta$, is twice differentiable with respect to $\theta$ and the functions $\frac{\partial}{\partial \theta_{i}} f_{X}^{-1}(\omega, \theta)$ and $\frac{\partial^{2}}{\partial \theta_{j} \partial \theta_{k}} f_{X}^{-1}(\omega, \theta)$ are continuous at all $(\omega, \theta), \omega \neq 0$, for $1 \leq i, j, k \leq 3$;

(iv) the functions $g_{i X}(\omega, \theta)$ for $1 \leq i \leq 3$ are symmetric about $\omega=0$ for $\omega \in(-\pi, \pi]$ and $\theta \in \Theta$;

(v) $g_{i X}(\omega, \theta) \in L_{1}((-\pi, \pi])$ for all $\theta \in \Theta$ and $1 \leq i \leq 3$;

(vi) $f_{X}(\omega, \theta) g_{i X}(\omega, \theta)$ for $1 \leq i \leq 3$ are in $L_{1}((-\pi, \pi])$ and $L_{2}((-\pi, \pi])$ for all $\theta \in \Theta$;

(vii) there exists a constant $0<k \leq 1$ such that $|\omega|^{k} f_{X}(\omega, \theta)$ is bounded and $\frac{g_{i X}(\omega, \theta)}{|\omega|^{k}}$ for $1 \leq i \leq 3$ are in $L_{2}((-\pi, \pi])$ for all $\theta \in \Theta$;

(viii) the matrix $\left\{\frac{\partial}{\partial \theta} \log \left(f_{X}(\omega, \theta)\right)\right\}\left\{\frac{\partial}{\partial \theta} \log \left(f_{X}(\omega, \theta)\right)\right\}^{\top}$ is in $L_{1}((-\pi, \pi]) \times \Theta_{1}$, where $\Theta_{1} \in \Theta$. In addition, the matrix is continuous uniformly in $\theta \in \Theta$ for each given $\omega \in(-\pi, \pi)$.

Assumption 2.1(i) assumes that $V(t)$ and $B(t)$ of model (2) are mutually independent. In other words, it implies that there is no leverage effect. In many cases, one may need to consider the case where the relationship between the returns and the volatility is asymmetric. In this case, one would need to allow some kind of dependence between $B(s)$ and $B_{1}(t)$. Sections $1,2.2,3$ and 4 of the original version of this paper by Casas and Gao (2006) give some discussion.

Assumption 2.1(ii)(iii) imposes some conditions to ensure the identifiability and existence of the spectral density function $f_{X}(\omega, \theta)$ and thus the Gaussian time series $\left\{X_{t}\right\}$. Assumption 2.2 requires that the spectral density function $f_{X}(\omega, \theta)$ needs to satisfy certain smoothness and differentiability conditions in order to verify conditions (A2) and (A3) of Heyde and Gay (1993). 
Both Assumptions 2.1 and 2.2 are necessary for us to establish the following asymptotic consistency results. The assumptions are justifiable when the form of $\psi(\omega, \theta)$ is specified. For example, when $\psi(\omega, \theta)=\frac{1}{\Gamma^{2}(1+\beta)}$, Assumptions 2.1 and 2.2 hold automatically. In this case, it is obvious

$$
\int_{-\pi}^{\pi} f_{X}(\omega, \theta) d \omega=4 \int_{-\pi}^{\pi}\left(\sum_{k=-\infty}^{\infty} \phi_{X}(\omega-2 k \pi, \theta)\right) d \omega=4 \int_{-\infty}^{\infty} \phi_{X}(\omega, \theta) d \omega<\infty .
$$

For the second part of Assumption 2.1(i), using the following decomposition

$$
\begin{aligned}
f_{X}(\omega, \theta) & =4 \frac{\psi(\omega, \theta) \sigma^{2}}{|\omega|^{2 \beta}\left(\omega^{2}+\alpha^{2}\right)}+4 \sum_{k=1}^{\infty} \frac{\psi(2 k \pi-\omega, \theta) \sigma^{2}}{|2 k \pi-\omega|^{2 \beta}\left((2 k \pi-\omega)^{2}+\alpha^{2}\right)} \\
& +4 \frac{\psi(2 k \pi+\omega, \theta) \sigma^{2}}{|2 k \pi+\omega|^{2 \beta}\left((2 k \pi+\omega)^{2}+\alpha^{2}\right)} \equiv f_{1}(\omega, \theta)+f_{2}(\omega, \theta)+f_{3}(\omega, \theta),
\end{aligned}
$$

we have

$$
\int_{-\pi}^{\pi} \log \left(f_{X}(\omega, \theta)\right) d \omega \geq \int_{-\pi}^{\pi} \log \left(f_{1}(\omega, \theta)\right) d \omega>-\infty
$$

when $\psi(\omega, \theta)$ is specified as $\psi(\omega, \theta)=\frac{1}{\Gamma^{2}(1+\beta)}$.

For the case of $\psi(\omega, \theta)=\frac{1}{\Gamma^{2}(1+\beta)}$, Assumption 2.2 may be justified similarly as in the proof of Lemma B.1 of Gao, et al. (2001). Instead of giving such detailed verification, we establish the following theorem.

TheOrem 2.1. Suppose that Assumptions 2.1 and 2.2 hold. Then

(i) $\tilde{\theta}_{T}$ is a strongly consistent estimator of $\theta_{0}$.

(ii) Furthermore, if the true value $\theta_{0}$ is in the interior of $\Theta_{0}$, then as $T \rightarrow \infty$

$$
\sqrt{T}\left(\tilde{\theta}_{T}-\theta_{0}\right) \rightarrow N\left(0, \Sigma^{-1}\left(\theta_{0}\right)\right)
$$

where

$$
\Sigma(\theta)=\frac{1}{4 \pi} \int_{-\pi}^{\pi}\left(\frac{\partial}{\partial \theta} \log (f(\omega, \theta))\right)\left(\frac{\partial}{\partial \theta} \log (f(\omega, \theta))\right)^{\top} d \omega,
$$

which is consistently estimated by $\widetilde{\Sigma}=\Sigma\left(\tilde{\theta}_{T}\right)$.

Similarly to some existing results (Heyde and Gay 1993; Robinson 1995; Hosoya 1997; Deo and Hurvich 2001; Gao 2004 and others), Theorem 2.1 shows that $\tilde{\theta}_{T}$ is still a $\sqrt{T}$-consistent estimator of $\theta_{0}$ even when $\left\{Z_{t}\right\}$ is non-Gaussian. In addition, we can use Theorem 2.1 to establish a consistent test statistic of the form

$$
L_{T}=T \tilde{\theta}_{T}^{\top} C^{\top}\left(C^{\top} \tilde{\Sigma}^{-1} C\right)^{-1} C \tilde{\theta}_{T}
$$

for testing the null hypothesis $H_{0}: C \theta_{0}=0$, where $C$ is an appropriate matrix. Theorem 2.1(ii) implies that $L_{T} \rightarrow_{D} \chi_{1}^{2}$ as $T \rightarrow \infty$. When the null hypothesis is true, 
it is an indication that one or more components of $\theta_{0}$ may be zero. The case where long-range dependence is absent can be included as a special case under $H_{0}$.

In Section 3 below, we apply our theory and estimation procedure to model (1). Our simulation results show that both the proposed theory and the estimation procedure work quite well numerically.

\section{Simulation Results}

Consider a simple model of the form

$$
d Y(t)=e^{X(t)} d B(t) \quad \text { with } \quad X(t)=\int_{-\infty}^{t} A(t-s) d B_{1}(s)
$$

where $A(x)=\frac{\sigma}{\Gamma(1+\beta)}\left(x^{\beta}-\alpha \int_{0}^{x} e^{-\alpha(x-u)} u^{\beta} d u\right)$. Note that $X(t)$ is a stationary version of $x(t)=\int_{0}^{t} A(t-s) d B_{1}(s)$, which is the solution of

$$
d x(t)=-\alpha x(t) d t+\sigma d B_{\beta}(t) .
$$

The stationary version of $x(t)$ has a spectral density function of the form

$$
\phi_{X}(\omega)=\phi_{X}(\omega, \theta)=\frac{\sigma^{2}}{\Gamma^{2}(1+\beta)} \frac{1}{|\omega|^{2 \beta}} \frac{1}{\omega^{2}+\alpha^{2}} .
$$

In order to implement our estimation procedure, we need to generate $\left\{X_{t}\right\}$ from such a Gaussian process with possible LRD. A relevant simulation procedure is given in Comte (1996), who proposes a discrete approximation to the solution of the continuous-time process $X(t)$. The addition of one more parameter does not provide additional information. Our simulation procedure based on the simulation of the covariance function of $X(t)$ is summarized as follows:

(i) Generate $C_{T}$, a $T \times T$ auto-covariance matrix, using the auto-covariance function given by $\gamma_{X}(\tau)=2 \int_{0}^{\infty} f_{X}(\omega, \theta) \cos (\omega \tau) d \omega$ with $\Delta=\frac{1}{250} . C_{T}$ is then a symmetric non-negative definite matrix with spectral decomposition $C_{T}=V \Lambda V^{\top}$, where $\Lambda=$ $\operatorname{diag}\left\{\lambda_{1}, \ldots, \lambda_{T}\right\}$ is the diagonal matrix of the eigenvalues and $V$ is the orthogonal matrix of the eigenvectors such that $V^{\top} V=I$ with $V^{\top}$ being the matrix transpose of $V$;

(ii) Generate a sample $G=\left(g_{1}, g_{2}, \ldots, g_{T}\right)^{\top}$ of independent realisations of a multivariate Gaussian random vector with the zero vector as the mean and the identity matrix as the covariance matrix; and

(iii) Generate $\left(X_{1}, \ldots, X_{T}\right)=V \Lambda^{1 / 2} V^{\top} G$ as the realization of a multivariate Gaussian random vector with the zero vector as the mean and $C_{T}$ as the covariance matrix.

The sample path of $\left\{X_{t}\right\}$ generated with the initial parameter values $\theta_{0}=(0.1,0.1,0.1)$ is illustrated in Figure 1. The periodogram $I_{N}^{\tilde{X}}$ and the spectral density $\phi_{\tilde{X}}$ of the simulated data set are illustrated in Figure 2. 
Figures 1 and 2 near here

We then generate $\left\{Z_{t}\right\}$ from (10) with $\Delta=\frac{1}{250}$. To consider all possible cases, the proposed estimation procedure was applied to the LRD case of $0<\beta<\frac{1}{2}$, the IRD case of $-\frac{1}{2}<\beta<0$ and the case of $\beta=0$. Sample sizes of $T=512,1024$ and 2048 were considered. The number of 400 replications was used for each case. The function (16) is symmetrical respect to $\omega$, this reduce the number of computational operations in half. Simulation results are displayed in Tables 1 and 2 below. The results in Tables 1-2 show the empirical means, the empirical standard deviations and the empirical mean squared errors (MSEs) of the estimators. The empirical mean of the absolute value of the corresponding estimated bias is given in each bracket beneath the corresponding estimator. Each of the MSEs is computed as a sum of two terms: the square of the estimated bias and variance.

Tables 1-2 near here

The first section of Table 1 provides the corresponding results for the cases where $\theta_{0}=$ $(0.8,0,0.5)$ and $\theta_{0}=(0.001,0,0.01)$. These results show that the estimation procedure works well for the SRD case where the initial parameter value for $\beta$ is zero as well as for small $\sigma_{0}$ and $\alpha_{0}$.

From the third section of Table 1 to Table 2, several different pairs of positive and negative $\beta$ values are considered. The corresponding results show that the MSEs for both $\alpha_{0}$ and $\sigma_{0}$ remain stable when $\beta_{0}$ changes from a positive value to its negative counterpart.

Individually, we have considered both relatively large and small values for $\beta$ to assess whether the estimation procedure is sensitive to the choice of $\beta$ values. These results in Table 1 show that there is some MSE distortion in the case of $T=512$ for $\beta_{0}$ when $\beta_{0}$ is as small as either 0.01 or -0.01 . When $T$ increases to 1024 and then 2048, the MSEs become stable. For the case of $\beta_{0}=0.45$ which appears in Table 3 , the MSEs look quite stable and very small.

In Table 2 , both relatively large and relatively small values for $\sigma_{0}$ have been considered. For the case of $\sigma_{0}=10$ in the first part of Table 2, the MSEs for the estimates of all the components of $\theta_{0}$ are quite stable and very small particularly when $T=2048$.

Since empirical financial evidence also suggests that very small volatility parameter values make precise estimation quite difficult, we also consider two cases in the second part of Table 2 that the volatility parameter value is as small as $\sigma_{0}=0.01$. These results 
show that the MSEs become quite reasonable even when the sample size is as medium as $T=512$. It is also observed from Table 2 that the change of the memory parameter value from $\beta_{0}=0.2$ to $\beta_{0}=-0.2$ does not affect the estimates of $\alpha_{0}$ and $\sigma_{0}$ significantly.

\section{Tables 3-4 near here}

The memory parameter $\beta$ is commonly estimated using the so-called GPH method proposed by Geweke and Porter-Hudak (1983) and then extended in Robinson (1995) and others. It has the property of being easily implemented and very fast. Deo and Hurvich (2001) study the bias and variance of this estimator for the memory parameter $\beta$ based on the fact that the periodogram of $Z_{1}, Z_{2}, \ldots, Z_{T}$ is calculated for certain Fourier frequencies $\omega_{j}=\frac{2 \pi j}{T}$ for $j=1,2, \ldots, m$, where $m$ is chosen such that $\frac{m}{T} \rightarrow \infty$ as $m \rightarrow \infty$ and $T \rightarrow \infty$. Discussion about the choice of $m$ may be found from Robinson (1994).

Tables 3-4 compare the memory parameter estimators derived from the Whittle procedure with the GPH estimators for $m=T^{0.3}$ and $m=T^{0.5}$. The motivation behind the choice of $m$ comes from Deo and Hurvich (2001) that study the GPH estimation method for $m=T^{0.3}, T^{0.4}$ and $T^{0.5}$. The GPH estimation method is appropriate for positive values of $\beta$ which are those displayed in these tables. As in Deo and Hurvich (2001), the bias of the estimator $\tilde{\beta}_{\mathrm{GPH}}$ increases when $m$ increases, however, the variance decreases as $m$ increases. In other words, the bias of the GPH estimator with $m=T^{0.5}$ is greater than the bias with $m=T^{0.3}$. However the MSE is smaller for $m=T^{0.5}$ than that for $m=T^{0.3}$ due to smaller variance in each case. As expected, the MSE decreases as the sample size $T$ increases.

Tables 3-4 show that the Whittle procedure performs better than the GPH method in all the cases under consideration. Our experience also shows that the computing time for using the GPH method is much shorter than that for the Whittle method. It is our opinion that the GPH method may not be applicable to estimate all the parameters $\alpha$, $\beta$ and $\sigma$ involved in the model as the estimation method was proposed to estimate the $\beta$ parameter only. Our study however shows that the proposed estimation method based on the Whittle method is applicable to the estimation of all the parameters and works well numerically in all the cases under consideration.

Regarding the asymptotic normality of the errors, Figures 3-4 show the density estimates of $\sqrt{T}\left(\tilde{\theta}_{T}-\theta_{0}\right)$ for $T=512,1024$ and 2048. It is clear that as $T$ increases the estimated density becomes closer to the density function of a Gaussian process with mean zero. The package KerSmooth in $\mathrm{R}$ was used to estimate the density function. 
Figures $3-4$ near here

In summary, the MSEs in Tables 1-4 demonstrate that both the proposed Whittle estimation procedure and the asymptotic convergence established in Theorem 2.1(i) work well numerically. Our preliminary simulation results further show that the MSEs become smaller when $\Delta=\frac{1}{250}$. In addition, Figures 3-4 show graphically the asymptotic normality of the errors established in Theorem 2.1(ii). In Section 4 below, both the proposed theory and the estimation procedure are also applied to several stock market indexes.

\section{Applications to Market Indexes}

Market indexes are a guideline of investor confidence and are inter-related to the performance of local and global economies. Investments on market indexes such as the Dow Jones, S\&P 500, FTSE 100, etc. are common practice. In this section, we apply model (20) to model the stochastic volatility and then the estimation procedure to assess both the memory and volatility properties of such indexes. Section 4.1 provides the explicit expressions of both the mean and the variance functions of stochastic volatility process $V(t)$. Section 4.2 describes briefly these indexes. Some empirical results are given in Section 4.3 below. Section 4.4 discusses a direct volatility estimation method.

\subsection{Estimation of mean and variance}

In addition to finding our estimates for the three parameters $\alpha, \beta$ and $\sigma$ involved in model (20), we are also interested to estimate both the mean $\mu_{V}$ and the standard deviation $\sigma_{V}$ of the stochastic volatility process $V(t)$.

Since we are concerned with the stationary version $V(t)=e^{X(t)}$ in model $(20)$, we are able to express both the mean and variance functions of $V(t)$ as follows:

$$
\mu_{V}=\mu_{V}(\theta)=\exp \left(\sigma_{X}^{2} / 2\right) \quad \text { and } \quad \sigma_{V}^{2}=\sigma_{V}^{2}(\theta)=\left(\exp \left(\sigma_{X}^{2}\right)-1\right) \exp \left(\sigma_{X}^{2}\right)
$$

where

$$
\sigma_{X}^{2}=\sigma_{X}^{2}(\vartheta)=\gamma_{X}(0)=\frac{\sigma^{2} \pi}{\Gamma^{2}(1+\beta) \alpha^{1+2 \beta} \cos (\beta \pi)} .
$$

We then estimate the mean $\mu_{V}(\theta)$ and the standard deviation $\sigma_{V}(\theta)$ by

$$
\tilde{\mu}_{V}=\mu_{V}\left(\tilde{\theta}_{T}\right) \quad \text { and } \quad \tilde{\sigma}_{V}=\sigma_{V}\left(\tilde{\theta}_{T}\right)
$$

with $\tilde{\theta}_{T}$ being defined before. For the market indexes, the corresponding estimates are given in Tables 6 and 7 below. We now provide some detailed descriptions about the data in Section 4.2 below. 


\subsection{Data}

The data sets chosen for our empirical study are the following daily recorded financial stock indexes: a) two major American indexes: Dow Jones Industrial Average (from the $1^{\text {st }}$ of October 1928 to the $29^{\text {th }}$ of July 2005) and S\&P 500 (from the $3^{\text {rd }}$ of January 1950 to the $29^{\text {th }}$ of July 2005); b) three of the major European indexes: CAC 40 (from the $30^{\text {th }}$ of December 1987 to the $29^{\text {th }}$ of July 2005), DAX 30 (from the $31^{\text {st }}$ of December 1964 to the $29^{\text {th }}$ of July 2005) and FTSE 100 (from the $31^{\text {st }}$ of January 1978 to the $29^{\text {th }}$ of July 2005); and c) the major Asian index, the NIKKEI 225 (from the $4^{\text {th }}$ of January 1984 to the $29^{\text {th }}$ of July 2005). A vast amount of information about market indexes is available on the Internet. Wikipedia and InvestorWords.com give easy access to informative glossaries of the stock market indexes. A figure of each of these indexes is given in Figure 5. The statistical summary of these financial series in Table 5 shows that the compounded returns series is stationary and has small standard deviation, small skewness and large kurtosis.

$$
\text { Figure } 5 \text { near here }
$$

\section{Table 5 near here}

\subsection{Empirical Results}

We have applied the proposed methodology to the real financial data. The results in Table 6 contain the estimates of $\alpha, \beta, \sigma, \mu_{V}$, and $\sigma_{V}$. The values in brackets correspond to the empirical MSEs showing that the asymptotic error is small in each case. The MSE values in Table 6 are indicative and calculated based on 400 replications with the sample size of $\mathrm{T}=2048$. These values are expected to be smaller for larger sets of data.

\section{Tables 6-7 near here}

Results of the estimation for the symmetric volatility case are displayed in Table 6 which shows that the DJIA and the S\&P 500 volatility processes display strong LRD. This result is expected from the studies of Ding et al. (1993) who show that the autocorrelation of the square of the returns of the S\&P 500 decrease at a very slow rate. However, the volatility process of the DJIA has a larger mean and a larger standard deviation. This difference is due to the difference in periods of the data sets as the DJIA was studied from 1928 and the S\&P 500 from 1950. The former set includes the data related to the Great Depression which influences the results. 
Results of Table 6 for the European indexes shows that the volatility of these indexes also displays LRD. The German DAX 30 has the largest mean and the largest standard deviation of the volatility process with values 1.3587 and 1.2499 respectively. In this respect, the CAC 40, the FTSE 100 and the S\&P 500 have very similar values for the mean (1.1458, 1.1955 and 1.1550 respectively) and standard variation (0.6410, 0.7833 and 0.6676 respectively). This suggests that the volatility process of these two European indexes and the S\&P 500 behave very similarly. In fact, the CAC 40 and FTSE 100 have a large percentage of shares belonging to foreign investors.

The long memory parameter estimate of the volatility process of the NIKKEI 225 has a value of 0.4860 , again showing a very strong LRD. The mean (2.0768) and standard deviation (3.7801) of its volatility process are significatively larger than those of the European and American indexes. This is not surprising, since the risky behaviour of the Japanese stock market is a well-known fact.

Results of Table 7 compare the estimation of the $\beta$ parameter using the Whittle method and the GPH method with $m=T^{0.3}, T^{0.4}$ and $T^{0.5}$. The GPH estimator also suggests that the volatility process of all the indexes displays some kind of LRD. Again, the choice of $m$ influences highly on the results.

\subsection{Volatility Estimation}

Estimating $\alpha, \beta$ and $\sigma$ is equivalent to estimating the covariance matrix of $\left\{X_{t}\right\}$ which the information necessary to be able to obtain an estimate of the volatility process using the Kalman filter presented in Harvey (1989) and applied to this particular problem in Harvey (1998). The estimate $\tilde{X}_{t}$ is given by,

$$
\widetilde{X}_{t}=\left(\mathbf{I}-\sigma_{e}^{2} C^{-1}\right) W_{t}+\sigma_{e}^{2} C^{-1} \mathbf{i} k,
$$

where $C=4 C_{X}+C_{e}$ with $C_{X}$ being the covariance matrix of $\left\{X_{t}\right\}$ and $C_{e}$ being the diagonal matrix with values $\sigma_{e}^{2}, \mathbf{I}$ is the identity matrix and $\mathbf{i}$ is a vector of 1 s and $k$ is estimated by the mean of $W_{t}$. Thus, $\widetilde{V}_{t}=\exp \left(\widetilde{X}_{t}\right)$. A comparison of this $\widetilde{V}_{t}$ with the absolute value of the compounded returns can be found in Figure 6 . The dotted line corresponds to $\left|W_{t}\right|$ and the read line corresponds to the volatility process estimator. The estimation has been done for the first 500 values of each of the indexes.

In summary, the volatility process of the DJIA, the S\&P 500 and the DAX 30 does display strong LRD for the symmetric volatility case. The CAC 40, the FTSE 100 and the NIKKEI 225 have a volatility with LRD for both the symmetric and asymmetric cases. In other words, the volatility processes of all these indexes present statistical 
patterns that repeat at different scales of time. Therefore, investments based on the prediction of the volatility process of each of these stock market indexes may exploit this fact.

\section{Discussion}

This paper has established a general class of stochastic volatility models with either LRD, IRD, or SRD. An estimation procedure has been proposed to deal with cases where a class of non-Gaussian processes may display LRD, IRD or SRD. We then have conducted some comprehensive simulation studies to show that the proposed estimation procedure works well numerically not only for the LRD parameter $\beta$, but also for both the drift parameter $\alpha$ and the volatility parameter $\sigma$. Our theory and estimation procedure has also been applied to estimate the volatility of some well-known stock market indexes.

As imposed in Assumption 2.1(i), we have assumed the mutual independence of the two Brownian motion processes in order to clearly present the main ideas as well as the estimation procedure. As briefly discussed in Casas and Gao (2006), in order to take leverage effects into account we would need to impose some kind of dependent structure on the covariance matrix of the two Brownian motion processes. As expected, such dependent structure would make the current discussion much more complicated. We thus wish to leave such an extension for future research.

\section{ACKNOWLEDGMENTS}

The authors would like to thank Michael McAleer and two referees as well as the participants of the 2006 International Conference on Time Series Econometrics, Finance and Risk in Perth for their constructive suggestions and comments. The first author would also like to thank Helena Veiga for her comments. Financial support from the Australian Research Council is acknowledged.

\section{Appendix A. Proof of Theorem 2.1}

This appendix provides only an outline of the proof of Theorem 2.1, since some technical details are quite standard but tedious in this kind of proof and therefore omitted here.

To prove Theorem 2.1, we need to introduce the following lemmas. 
Lemma A.1. Suppose that Assumptions 2.1 and 2.2 hold. Then for every continuous function $w(\omega, \theta)$

$$
\int_{-\pi}^{\pi} I_{T}(\omega) w(\omega, \theta) d \omega \rightarrow \int_{-\pi}^{\pi} f\left(\omega, \theta_{0}\right) w(\omega, \theta) d \omega
$$

with probability one as $T \rightarrow \infty$.

Proof: The conclusion in (27) is the same as Lemma 1 of Fox and Taqqu (1986) for the cases where the process involved is Gaussian. The proof of their Lemma 1 basically follows that of Lemma 1 of Hannan (1973), which does not impose any Gaussian assumptions on the process involved.

In view of the expression of $Z_{t}=\mu+2 X_{t}+e_{t}$ in (10) as well as Lemma 1 of Hannan (1973), therefore the proof of Lemma 1 of Fox and Taqqu (1986) remains valid for the case where $\left\{X_{t}\right\}$ is the Gaussian time series with LRD, $\left\{e_{t}\right\}$ is a sequence i.i.d. random errors and thus $\left\{Z_{t}\right\}$ is non-Gaussian. This is mainly because of the following two reasons.

The first reason is that Assumption 2.1(ii) guarantees that $\left\{X_{t}\right\}$ admits a backward expansion of the form (see Fox and Taqqu 1986, p.520)

$$
X_{t}=\sum_{s=0}^{\infty} b_{s} u_{t-s}
$$

where $\left\{b_{s}\right\}$ is a sequence of suitable real numbers such that $\left\{X_{t}\right\}$ is a Gaussian process with its spectral density function being given by $f_{X}(\omega, \theta)$, and $\left\{u_{s}:-\infty<s<\infty\right\}$ is a sequence of independent and normally distributed random variables with $E\left[u_{s}\right]=0$ and $E\left[u_{s}^{2}\right]=1$. Thus, $\left\{Z_{t}\right\}$ can be written into a partial sum of independent random variables of the form

$$
Z_{t}=\mu+e_{t}+2 \sum_{s=0}^{\infty} b_{s} u_{t-s} .
$$

The second reason is that Lemma 1 of Hannan (1973) is applicable to non-Gaussian time series and thus to $\left\{Z_{t}\right\}$ of the form (29).

Lemma A.2. Suppose that Assumptions 2.1 and 2.2 hold. Then as $T \rightarrow \infty$

$$
W_{T}(\theta) \rightarrow W(\theta)=\frac{1}{4 \pi} \int_{-\pi}^{\pi}\left\{\log (f(\omega, \theta))+\frac{f\left(\omega, \theta_{0}\right)}{f(\omega, \theta)}\right\} d \omega .
$$

Proof: The proof of (30) follows from (27).

Lemma A.3. Suppose that Assumptions 2.1 and 2.2 hold. Then as $T \rightarrow \infty$

$$
\tilde{\theta}-\theta_{0} \rightarrow 0 \quad \text { with probability one }
$$


Proof: Lemma A.2 implies that the following holds with probability one for $\theta \neq \theta_{0}$,

$$
W_{T}(\theta)-W_{T}\left(\theta_{0}\right) \rightarrow K\left(\theta, \theta_{0}\right)=\frac{1}{4 \pi} \int_{-\pi}^{\pi}\left\{\frac{f\left(\omega, \theta_{0}\right)}{f(\omega, \theta)}-1-\log \left(\frac{f\left(\omega, \theta_{0}\right)}{f(\omega, \theta)}\right)\right\} d \omega>0
$$

as $T \rightarrow \infty$.

Thus, for any given $\epsilon>0$

$$
\lim \inf _{T \rightarrow \infty} \inf _{\left\|\theta-\theta_{0}\right\| \geq \epsilon}\left(W_{T}(\theta)-W_{T}\left(\theta_{0}\right)\right)>0
$$

with probability one. The proof of $\tilde{\theta}_{T} \rightarrow \tilde{\theta}_{0}$ with probability one follows from Assumption 2.1(iii). Thus, the proof of Lemma A.3 is finished.

Proof of TheOrem 2.1(i): The first part of Theorem 2.1 has already been proved in Lemma A.3.

Proof of Theorem 2.1(ii): The proof of the second part of Theorem 2.1 is a direct application of Theorem 1(ii) of Heyde and Gay (1993). Note that Assumption 2.1 implies that Condition (A1) of Heyde and Gay (1993) is satisfied because of (28) and the expression of $Z_{t}=\mu+4 X_{t}+e_{t}$. Assumption 2.2 implies that conditions (A2) and (A3) of Heyde and Gay (1993) are satisfied for $f_{X}(\omega, \theta)$ and then $f_{Z}(\omega, \theta)$. Thus, by applying the Mean-Value Theorem and Theorem 1(ii) of Heyde and Gay (1993), the proof of the asymptotic normality is completed.

Using Assumption 2.2(viii) and Theorem 2.1(i), the proof of $\Sigma\left(\tilde{\theta}_{T}\right) \rightarrow \Sigma\left(\theta_{0}\right)$ follows as $T \rightarrow \infty$.

\section{REFERENCES}

Andersen, T. G., And Lund, J. (1997), "Estimating Continuous-Time Stochastic Volatility Models of the Short Term Interest Rate", Journal of Econometrics, 77, 343-378.

Andersen, T. G., And Sørensen, B. E. (1996), "GMM Estimation of a Stochastic Volatility Model: A Monte Carlo Study", Journal of Business and Economic Statistics, 14, 328-352.

Andrews, D. W. K., And Sun, Y. (2004), "Adaptive Local Polynomial Whittle Estimation of LongRange Dependence", Econometrica, 72, 569-614.

Anh, V., And Heyde, C. (ed.) (1999), "Special Issue on Long-Range Dependence", Journal of Statistical Planning $\&$ Inference, 80, 1.

Anh, V., Heyde, C., And Leonenko, N. (2002), "Dynamic Models of Long Memory Processes Driven by Lévy Noise", Journal of Applied Probability, 39, 730-747.

Anh, V., And Inoue, A. (2005), "Financial Markets with Memory I: Dynamic Models", Stochastic Analysis 83 Its Applications, 23, 275-300.

Anh, V., Inoue, A., And Kasahara, Y. (2005), "Financial Markets with Memory II: Innovation 
Processes and Expected Utility Maximization", Stochastic Analysis \&3 Its Applications, 23, 301-328. Anh, V., Leonenko, N. N., Sakhno, L. M. (2004). "On a Class of Minimum Contrast Estimators for Fractional Stochastic Processes and Fields", Journal of Statistical Planning and Inference, 123, 161-185.

Arapis, M., And GaO, J. (2006), "Empirical Comparisons in Short-Term Interest Rate Models using Nonparametric Methods", Journal of Financial Econometrics, 4, 310-345.

Asai, M., McAleer, M., ANd Yu, J. (2006), "Multivariate Stochastic Volatility: a review", Econometric Reviews, 25, 145-175.

Baillie, R., And King, M. L. (ed.) (1996), "Special Issue of Journal of Econometrics", Annals of Econometrics, 73, 1.

Barndorff-Nielsen, O. E., And Leonenko, N. N. (2005). "Spectral Properties of Superpositions of Ornstein-Uhlenbeck Type Processes", Methodology and Computing in Applied Probability, 7, 335-352. Beran, J. (1994), Statistics for Long-Memory Processes, New York: Chapman and Hall.

Black, F., and Scholes, M. (1973), "The Pricing of Options and Corporate Liabilities", Journal of Political Economy, 3, 637-654.

Breidt, F. J., Crato, N., and de Lima, P. J. F. (1998), "The Detection and Estimation of LongMemory in Stochastic Volatility", Journal of Econometrics, 83, 325-348.

Brockwell, P., And Davis, R. (1990), Time Series: Theory and Methods, New York: Springer. Casas, I., And GaO, J. (2006), "Econometric Estimation in Stochastic Volatility Models with LongRange Dependence," Working paper available at http://www.maths.uwa.edu.au/ jiti/casgaolrd.pdf. Comte, F. (1996), "Simulation and Estimation of Long Memory Continuous Time Models", Journal of Time Series Analysis, 17, 19-36.

Comte, F., And Renault, E. (1996), "Long Memory Continuous-Time Models", Journal of Econometrics, 73, 101-150.

Comte, F., And Renault, E. (1998), "Long Memory in Continuous-Time Stochastic Volatility Models", Mathematical Finance, 8, 291-323.

Cox, J. C., Ingersoll, J.E. And Ross, S.A. (1985), "A Theory of the Term Structure of Interest Rates," Econometrica, 53, 385-407.

Deo, R., And Hurvich, C. M. (2001), "On the Log-Periodogram Regression Estimator of the Memory Parameter in Long Memory Stochastic Volatility Models", Econometric Theory, 17, 686-710.

Ding, Z., Granger, C. W. J., And Engle, R. (1993), "A Long Memory Property of Stock Market Returns and a New Model," Journal of Empirical Finance, 1, 83-105.

Fan, J., And Zhang, C. M. (2003), "A Re-examination of Stanton's Diffusion Estimators with Applications to Financial Model Validation." Journal of the American Statistical Association, 461, 118-134.

Fox, R., And Taquu, M.S. (1986), "Large-Sample Properties of Parameter Estimates for Strongly Dependent Stationary Gaussian Time Series", Annals of Statistics, 14, 512-532.

GaO, J. (2004), "Modelling Long-Range Dependent Gaussian Processes with Application in ContinuousTime Financial Models", Journal of Applied Probability, 41, 467-482.

Gao, J., Anh, V., And Heyde, C. (2002), "Statistical Estimation of Nonstationary Gaussian Processes with Long-Range Dependence and Intermittency", Stochastic Processes 83 Their Applications, 99, 295-321.

Gao, J., Anh, V., Heyde, C., And Tieng, Q. (2001), "Parameter Estimation of Stochastic Processes 
with Long-Range Dependence and Intermittency", Journal of Time Series Analysis, 22, 517-535.

Geweke, J., And Porter-Hudak, S. (1983), "The Estimation and Application of Long Memory Time Series Models", Journal of Time Series Analysis, 4, 221-238.

Hannan, E. J. (1973), "The Asymptotic Theory of Linear Time-Series Models", Journal of Applied Probability, 10, 130-145.

Harvey, A. C. (1989), Forecasting, Structural Time Series Models and the Kalman Filter, New York: Cambridge University Press.

Harvey, A. C. (1998), "Long Memory in Stochastic Volatility", in Knight, J. and Satchell, S. , Forecasting Volatility in the Financial Markets, Oxford: Butterworth and Heinemann.

Heyde, C., And Gay, R. (1993), "Smoothed Periodogram Asymptotics and Estimation for Processes and Fields with Possible Long-Range Dependence", Stochastic Processes and Their Applications, 45, 169-182.

Hosoya, Y. (1997), "A Limit Theory for Long-Range Dependence and Statistical Inference on Related Models", Annals of Statistics, 25, 105-137.

Hull, J., And White, A. (1987), "The Pricing of Options on Assets with Stochastic Volatilities", Journal of Finance, 2, 281-300.

Leonenko, N. N. and Sakhno, L. M. (2006). "On the Whittle Estimators for Some Classes of Continuous Parameter Random Processes and Fields", Statistics and Probability Letters, 76, 781-795.

Priestley, M. B. (1981), Spectral Analysis and Time Series, New York: Academic Press.

Robinson, P. (1994), "Rates of Convergence and Optimal Spectral Bandwidth for Long Range Dependence", Probability Theory and Related Fields, 99, 443-473.

Robinson, P. (1995), "Gaussian Semiparametric Estimation of Long-Range Dependence", Annals of Statistics, 23, 1630-1661.

Robinson, P. M. (ed.) (2003), "Time Series with Long Memory", Advanced Texts in Econometrics, Oxford: Oxford University Press.

Stoyanov, J. (2004), "Stieltjes Classes for Moment-Indeterminate Probability Distributions", Journal of Applied Probability, 41A, 281-294.

Sun, Y., And Phillips, P. C. B. (2003), "Nonlinear Log-periodogram Regression for Perturbed Fractional Processes", Journal of Econometrics, 115, 355-389.

Taylor, S. J. (1986), Modelling Financial Time Series, Chichester. U.K.: John Wiley.

TAYLOR, S. J. (1994), "Modelling Stochastic Volatility: A Review and Comparative Study", Mathematical Finance, 4, 183-204.

VAsicek, O. (1977), "An Equilibrium Characterization of the Term Structure", Journal of Financial Economics, 5, 177-188.

Whittle, P. (1951), Hypothesis Testing in Time Series Analysis, New York: Hafner.

\section{Appendix B. Tables and Figures}

This appendix lists all Tables and Figures for Sections 3 and 4. 


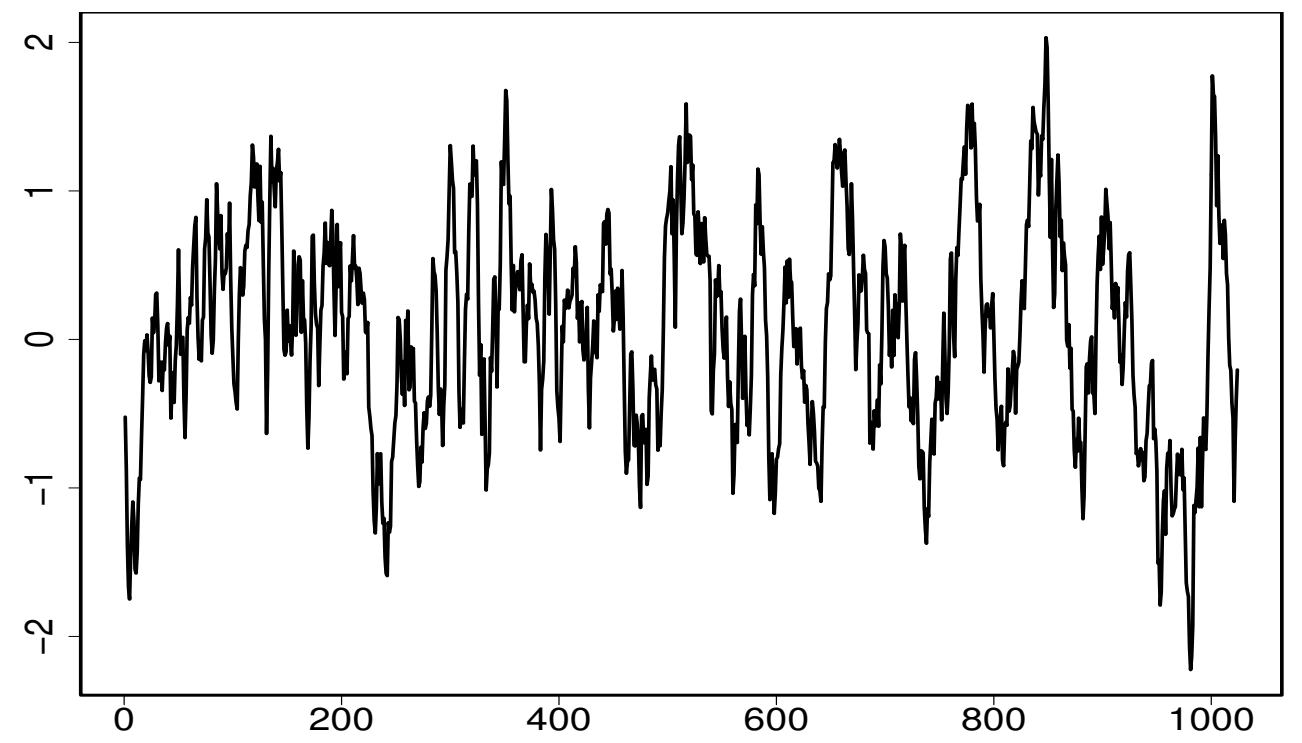

Figure 1: Sample path for data generated with $\theta_{0}=(0.1,0.1,0.1)$ and $\Delta=1$.

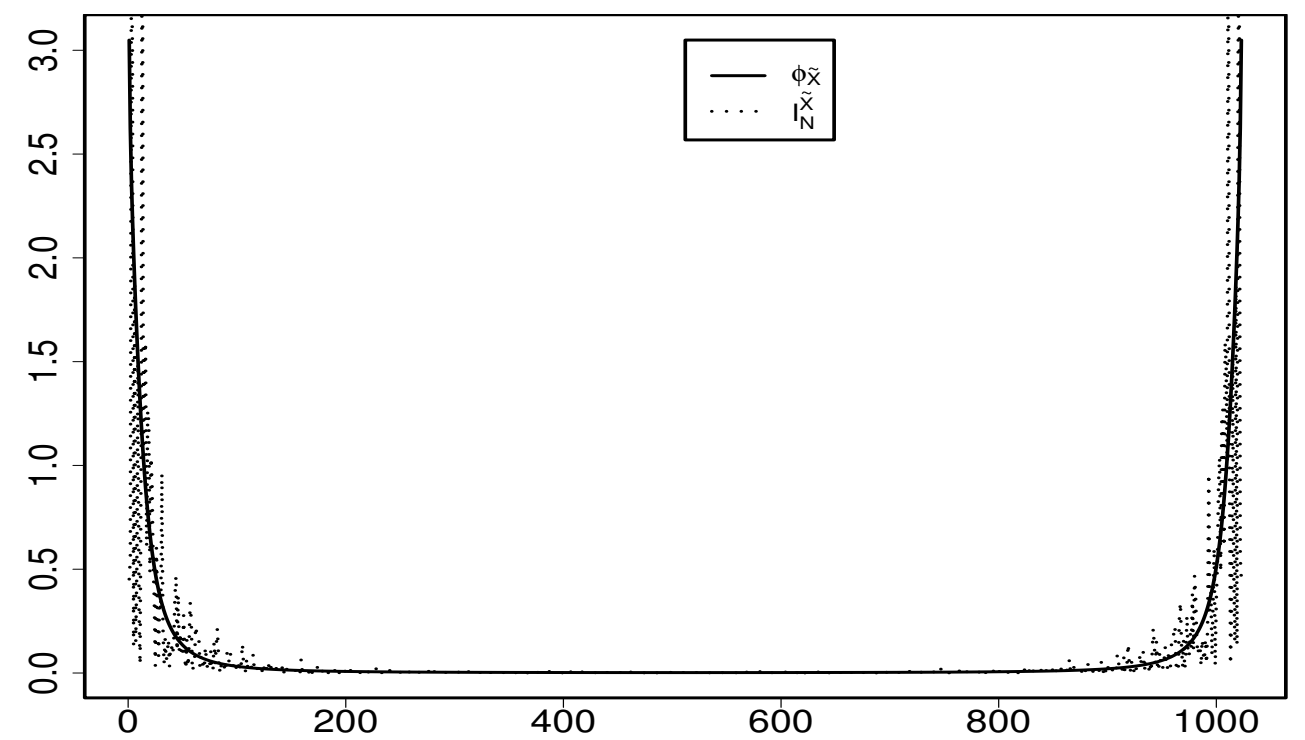

Figure 2: The periodogram and the spectral density for $\theta_{0}=(0.1,0.1,0.1)$. 


\begin{tabular}{|c|c|}
\hline 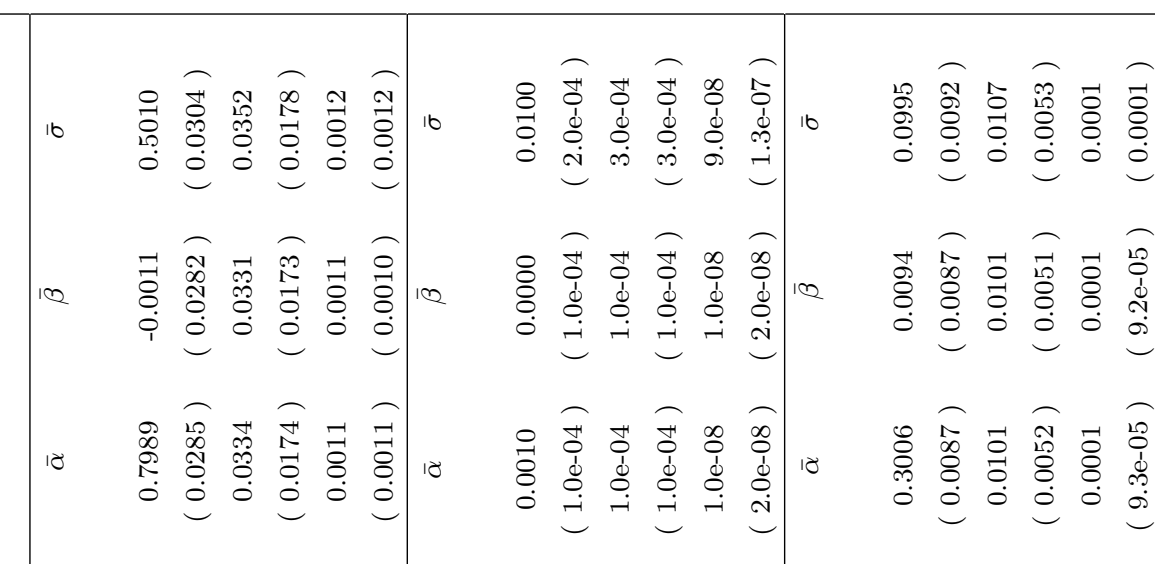 & 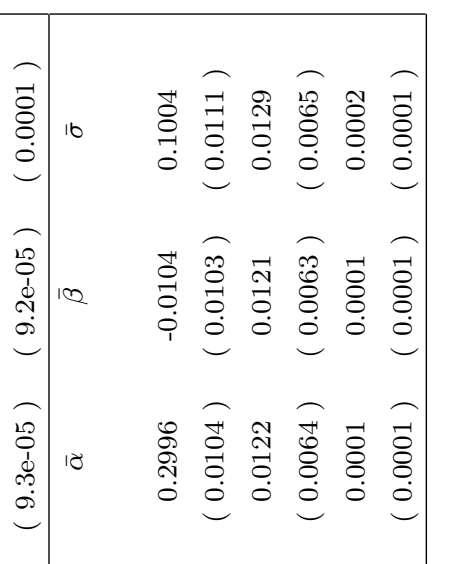 \\
\hline 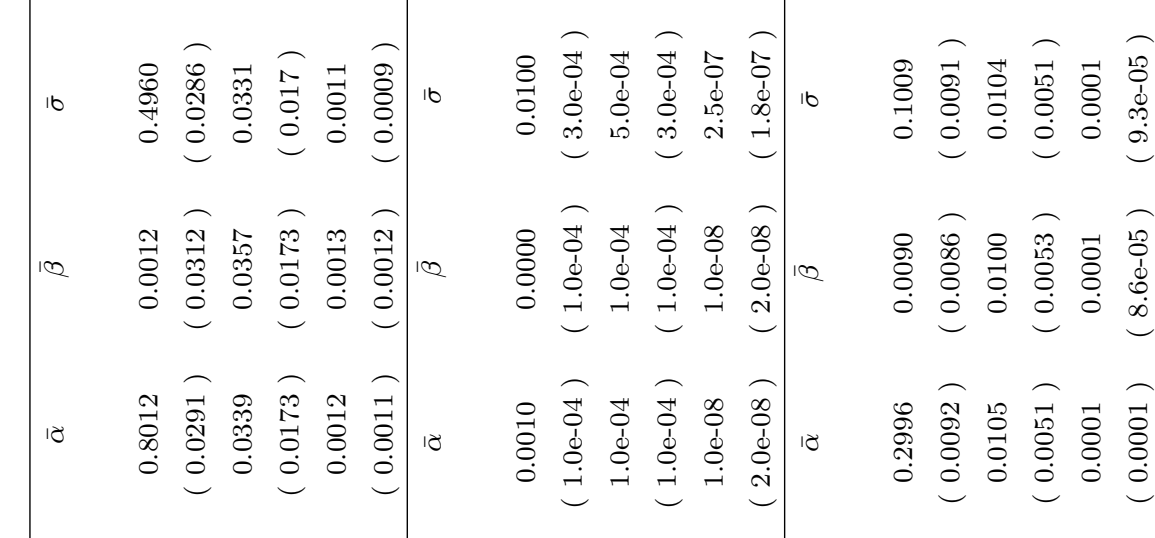 & 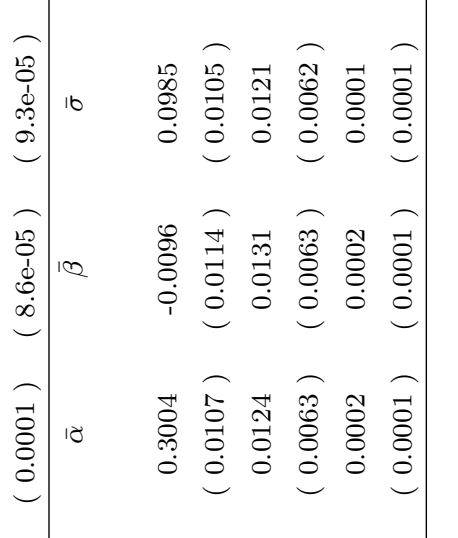 \\
\hline 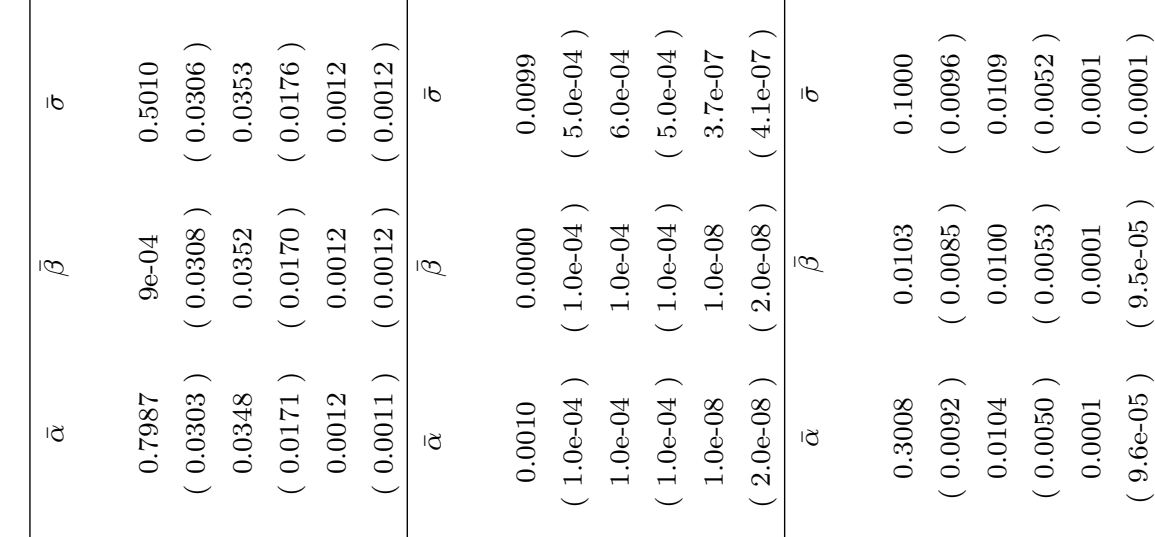 & 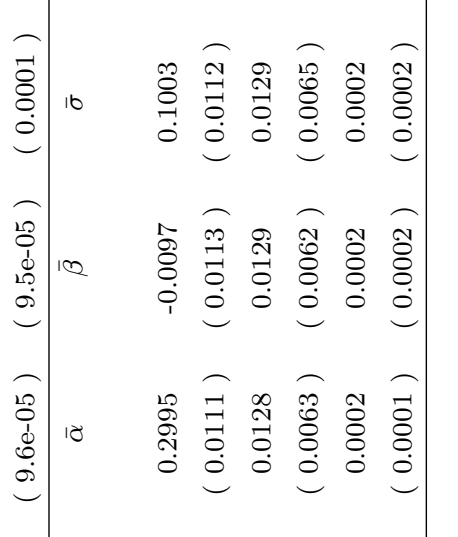 \\
\hline
\end{tabular}




\begin{tabular}{|c|c|}
\hline 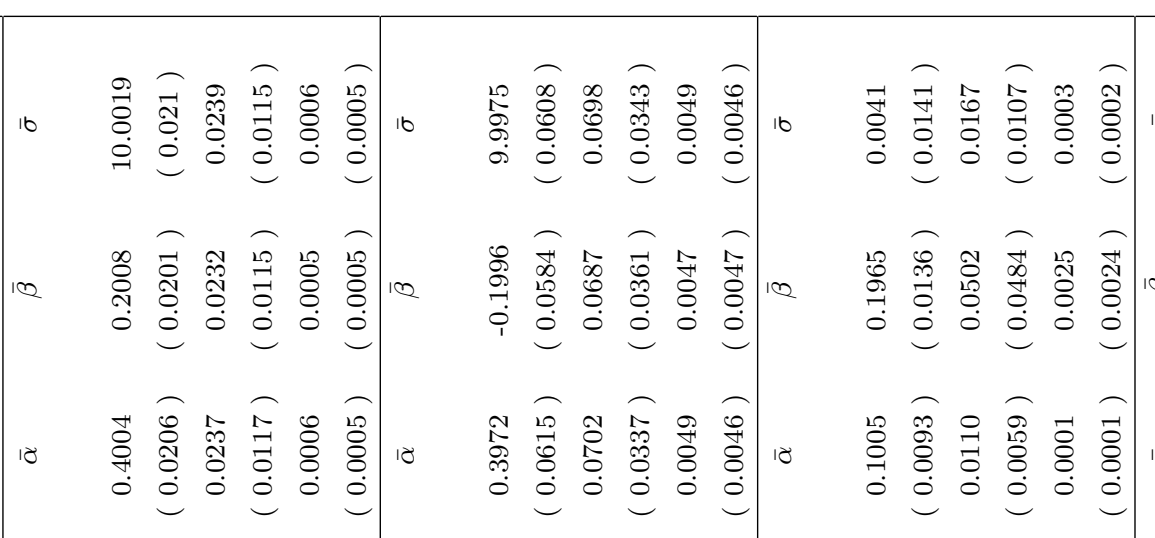 & 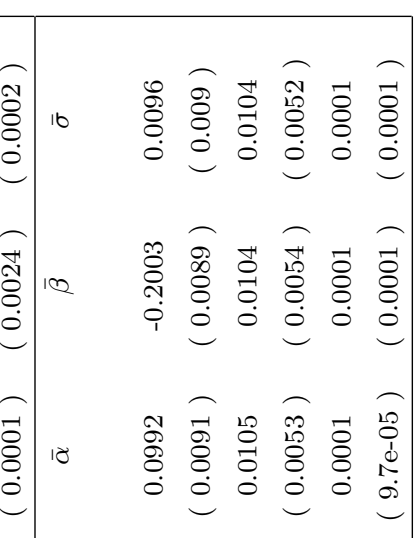 \\
\hline 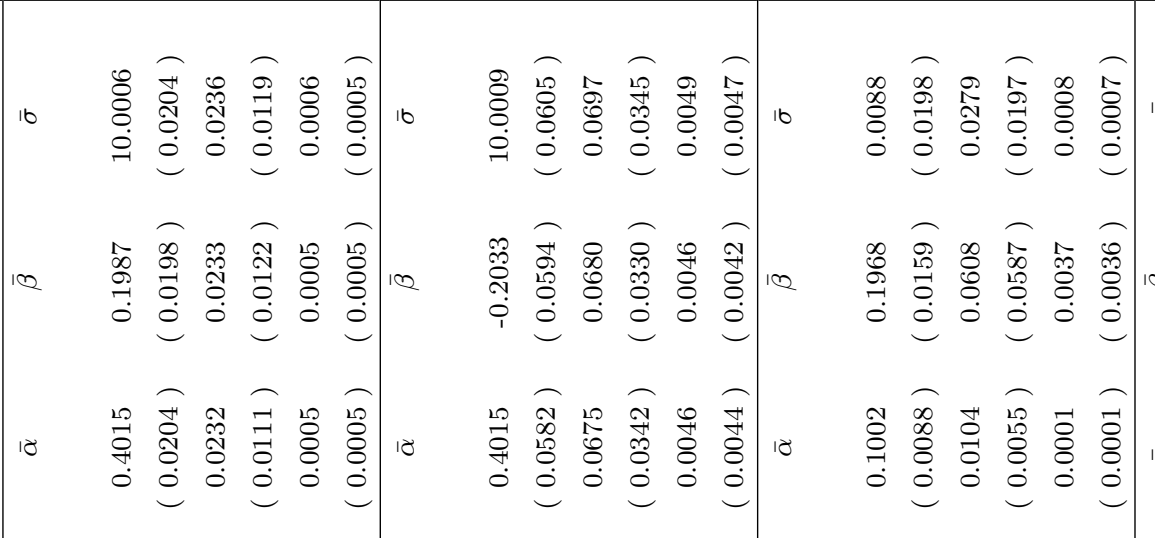 & 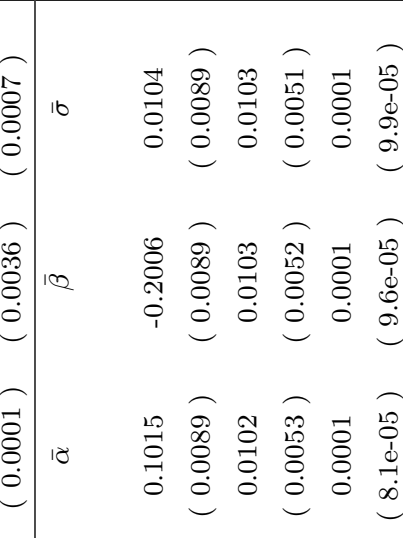 \\
\hline 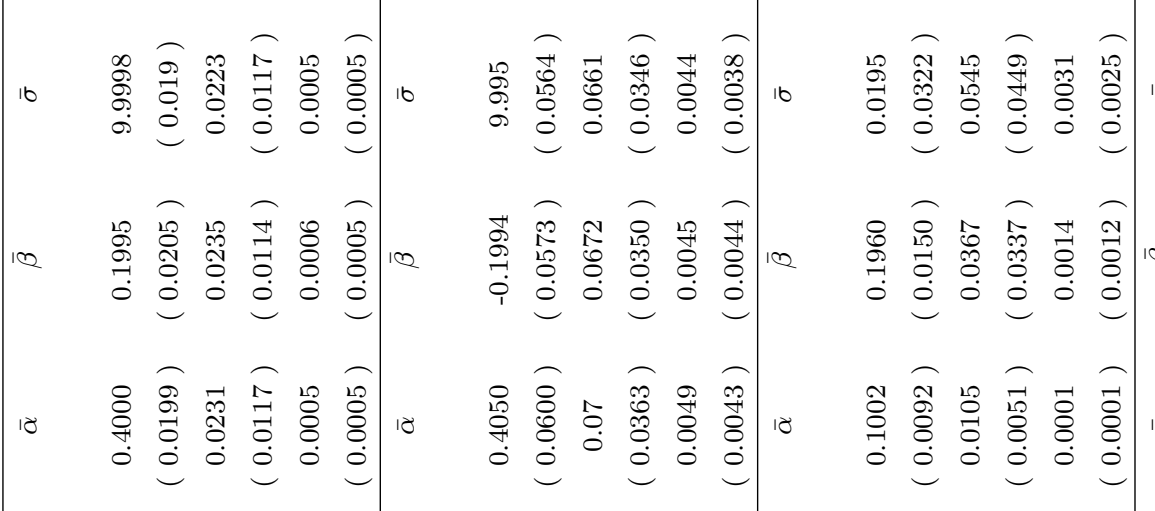 & 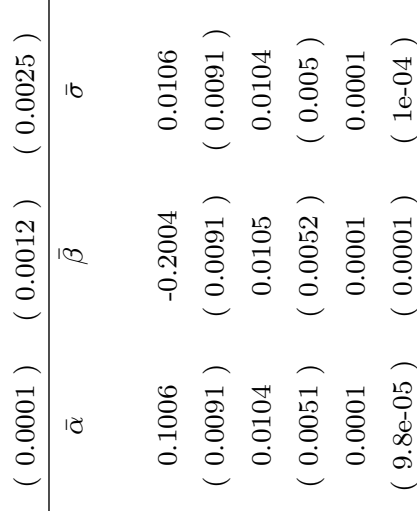 \\
\hline
\end{tabular}




\begin{tabular}{|c|c|c|c|}
\hline 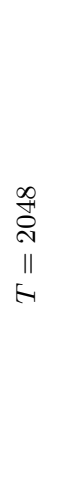 & 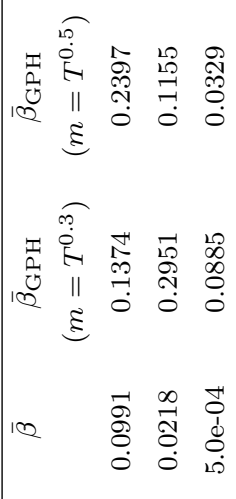 & 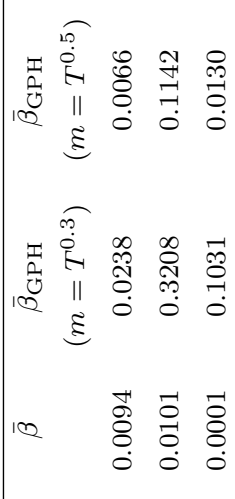 & 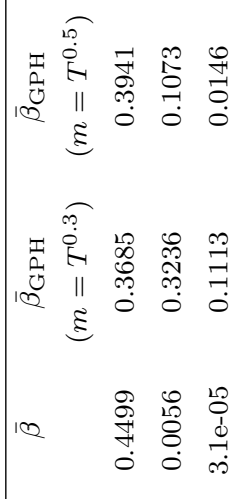 \\
\hline 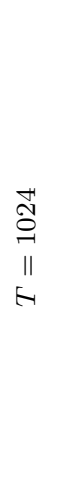 & 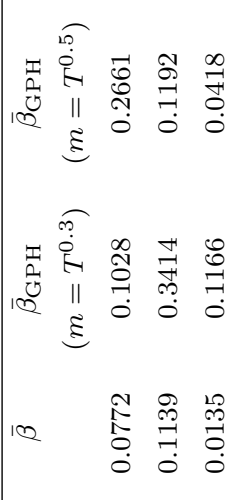 & 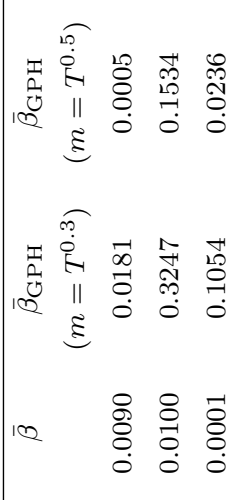 & 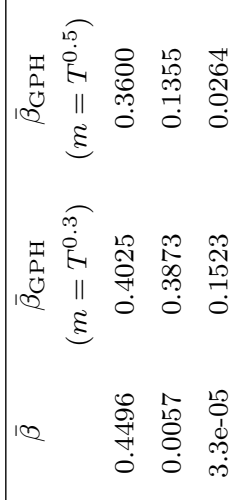 \\
\hline $\begin{array}{c}\text { II } \\
\text { ID } \\
11 \\
\text { I- }\end{array}$ & 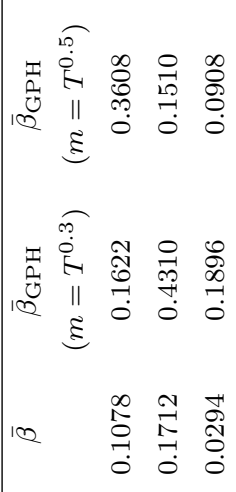 & 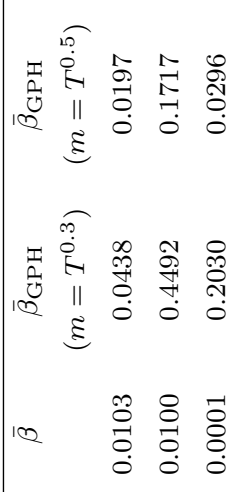 & 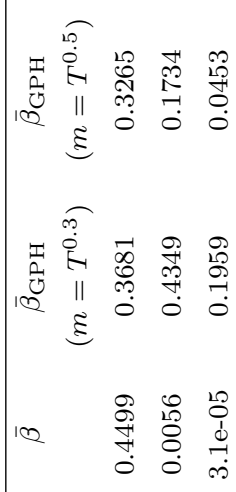 \\
\hline & 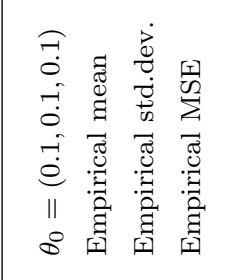 & 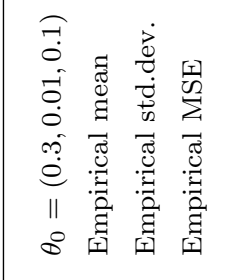 & 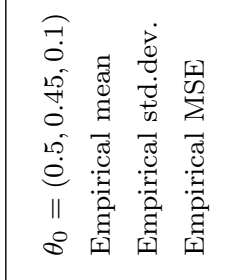 \\
\hline
\end{tabular}




\begin{tabular}{|c|c|c|c|}
\hline 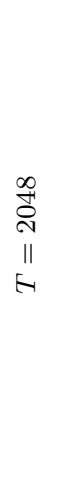 & 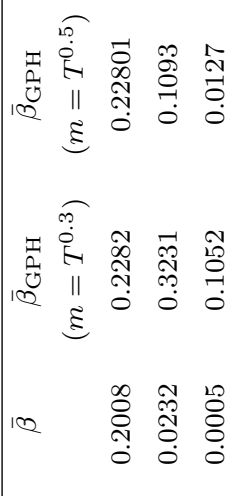 & 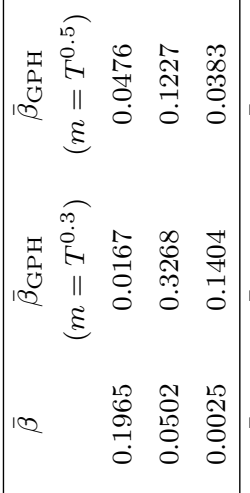 & 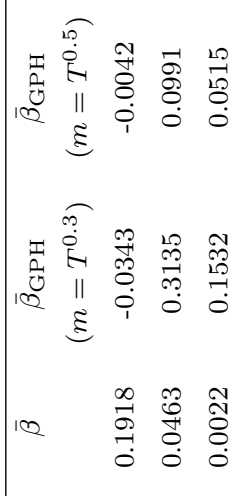 \\
\hline 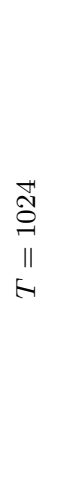 & 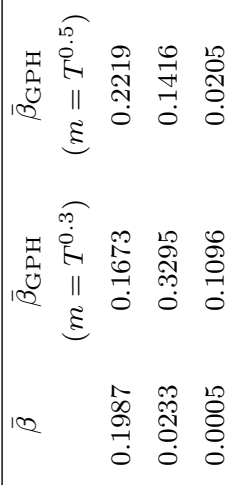 & 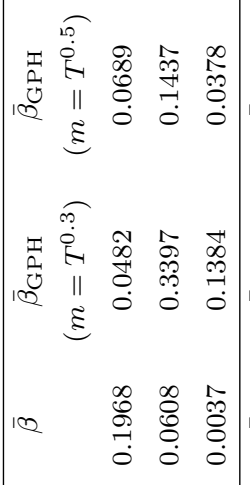 & 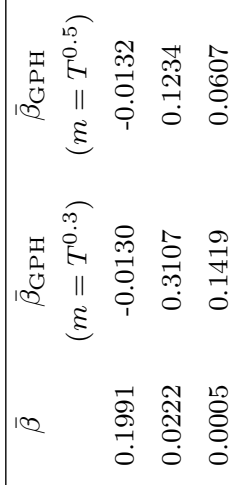 \\
\hline $\begin{array}{l}\text { Iี } \\
\text { ID } \\
11 \\
\text { H- }\end{array}$ & 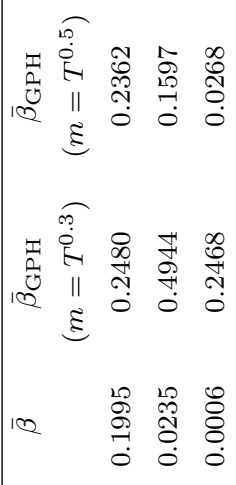 & 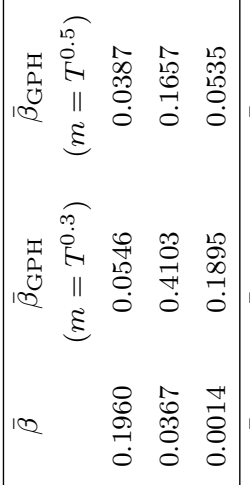 & 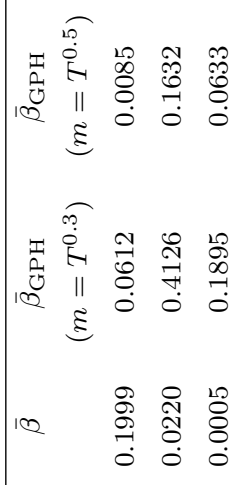 \\
\hline & 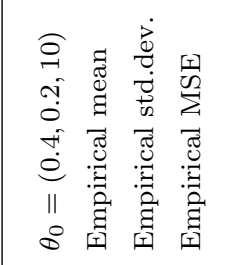 & 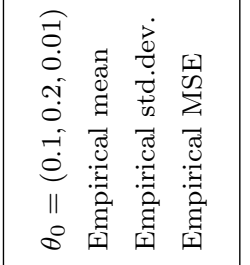 & 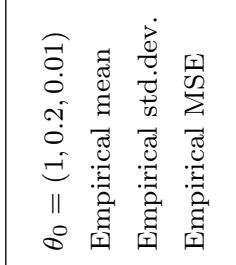 \\
\hline
\end{tabular}




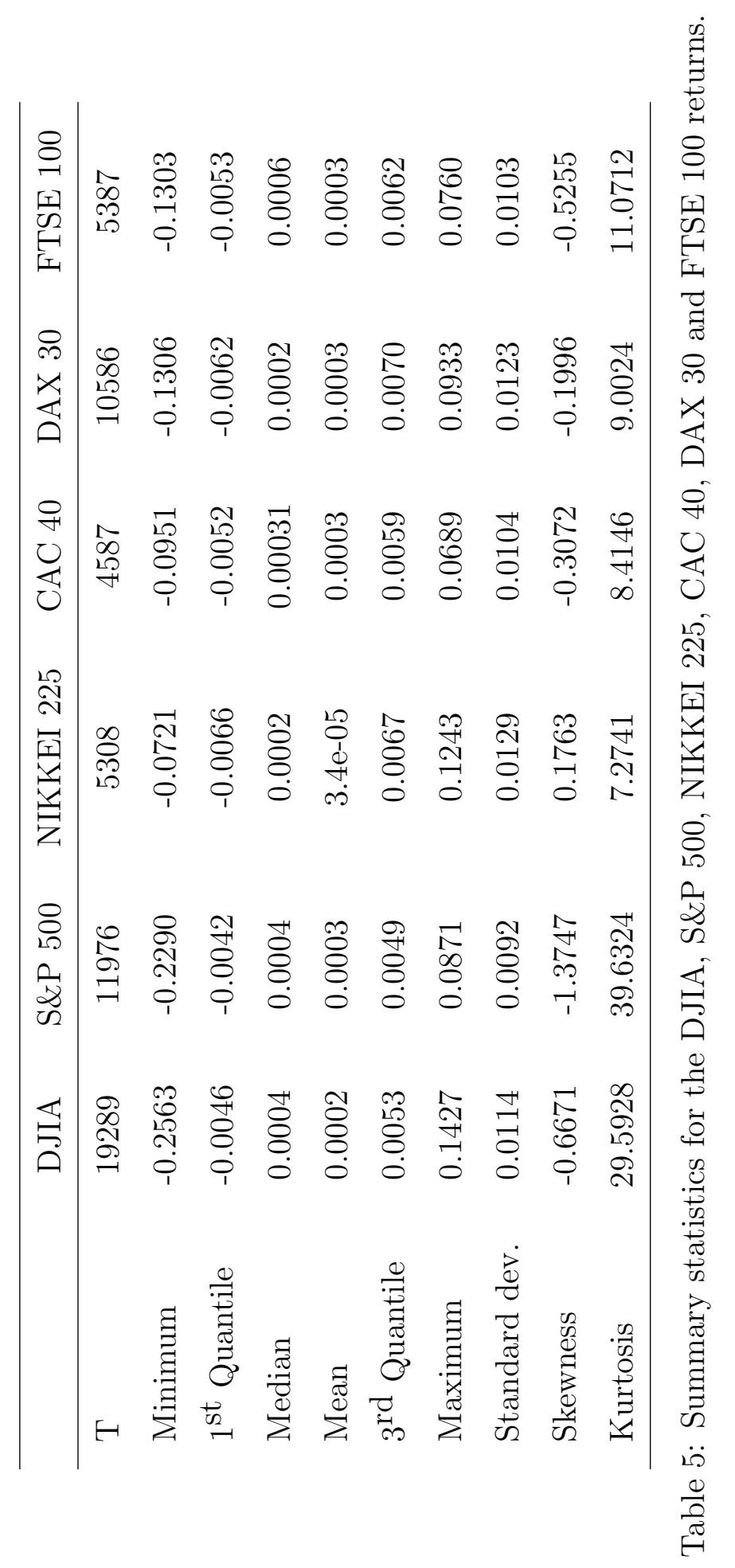




\begin{tabular}{|l|ccccc|}
\hline Index & $\bar{\alpha}$ & $\bar{\beta}$ & $\bar{\sigma}$ & $\bar{\mu}_{V}$ & $\bar{\sigma}_{V}$ \\
\hline DJIA & 0.0478 & 0.4783 & 0.0055 & 1.3482 & 1.2191 \\
S\&P 500 & $(2.7 \mathrm{e}-05)$ & $(3.1 \mathrm{e}-0.5)$ & $(1.7 \mathrm{e}-05)$ & & \\
& 0.0732 & 0.4563 & 0.0086 & 1.1550 & 0.6676 \\
CAC 40 & $(3.3 \mathrm{e}-05)$ & $(3.6 \mathrm{e}-05)$ & $(2.0 \mathrm{e}-05)$ & & \\
& 0.2122 & 0.4549 & 0.0237 & 1.1458 & 0.6410 \\
DAX 30 & $(0.0004)$ & $(0.0005)$ & $(0.0002)$ & & \\
FTSE 100 & 0.0877 & 0.4841 & 0.0085 & 1.3587 & 1.2499 \\
& $(9.8 \mathrm{e}-05)$ & $(0.0001)$ & $(5.5 \mathrm{e}-05)$ & & \\
NIKKEI 225 & 0.0588 & 0.4608 & 0.0073 & 1.1955 & 0.7833 \\
& $(2.4 \mathrm{e}-05)$ & $(2.7 \mathrm{e}-05)$ & $(1.9 \mathrm{e}-05)$ & & \\
& 0.0830 & 0.4860 & 0.0115 & 2.0768 & 3.7801 \\
& $(7.1 \mathrm{e}-05)$ & $(7.9 \mathrm{e}-05)$ & $(5.2 \mathrm{e}-05)$ & & \\
\hline
\end{tabular}

Table 6: Estimates of market indexes volatility parameters when independence between the two Brownian motions is assumed. The empirical MSE of the estimators is displayed in brackets.

\begin{tabular}{|l|cccc|}
\hline Index & $\bar{\beta}_{\mathrm{GPH}}\left(m=n^{0.3}\right)$ & $\bar{\beta}_{\mathrm{GPH}}\left(m=n^{0.4}\right)$ & $\bar{\beta}_{\mathrm{GPH}}\left(m=n^{0.5}\right)$ & $\bar{\beta}$ \\
\hline DJIA & 0.5772 & 0.7033 & 0.5496 & 0.4783 \\
S\&P 500 & 0.4892 & 0.5554 & 0.4929 & 0.4563 \\
CAC 40 & 0.3456 & 0.2319 & 0.3257 & 0.4549 \\
DAX 30 & 0.6505 & 0.6300 & 0.3620 & 0.4841 \\
FTSE 100 & 0.4805 & 0.5034 & 0.5462 & 0.4608 \\
NIKKEI 225 & 0.4522 & 0.4817 & 0.3929 & 0.4860 \\
\hline
\end{tabular}

Table 7: Comparison of the estimation of the LRD parameter of financial market indexes with the log-periodogram and the Whittle methods. 


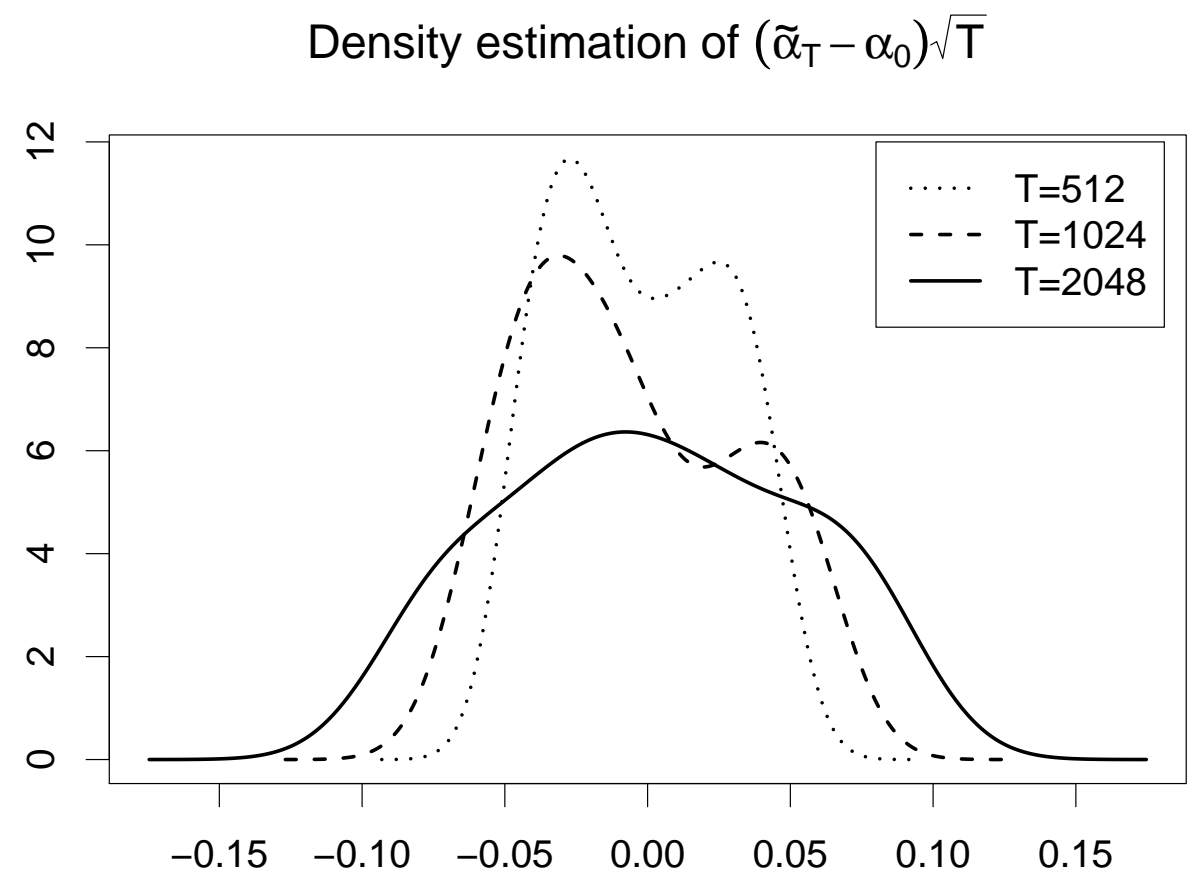

Density estimation of $\left(\tilde{\beta}_{T}-\beta_{0}\right) \sqrt{T}$

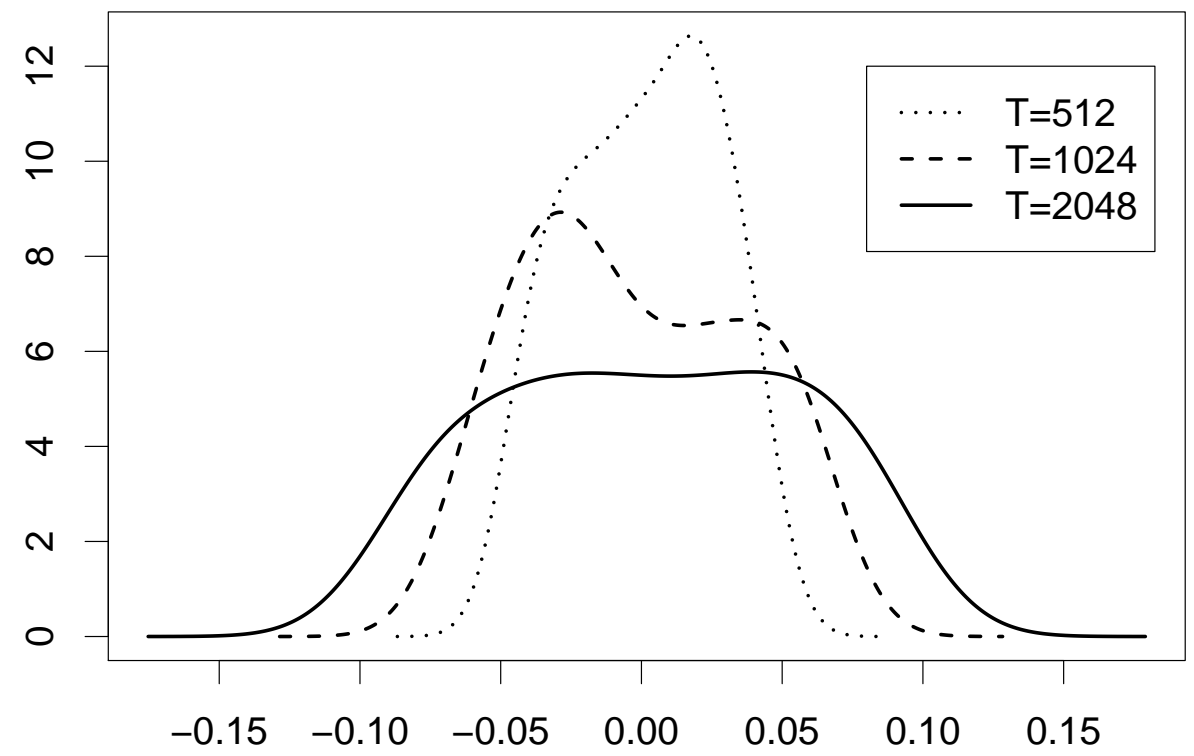

Figure 3: Density function estimates of $\sqrt{T}\left(\bar{\alpha}_{T}-\alpha_{0}\right)$ and $\sqrt{T}\left(\bar{\beta}_{T}-\beta_{0}\right)$ for $\theta_{0}=$ $\left(\alpha_{0}, \beta_{0}, \sigma_{0}\right)=(0.1,0.1,0.1)$. 
Density estimation of $\left(\tilde{\sigma}_{T}-\sigma_{0}\right) \sqrt{T}$

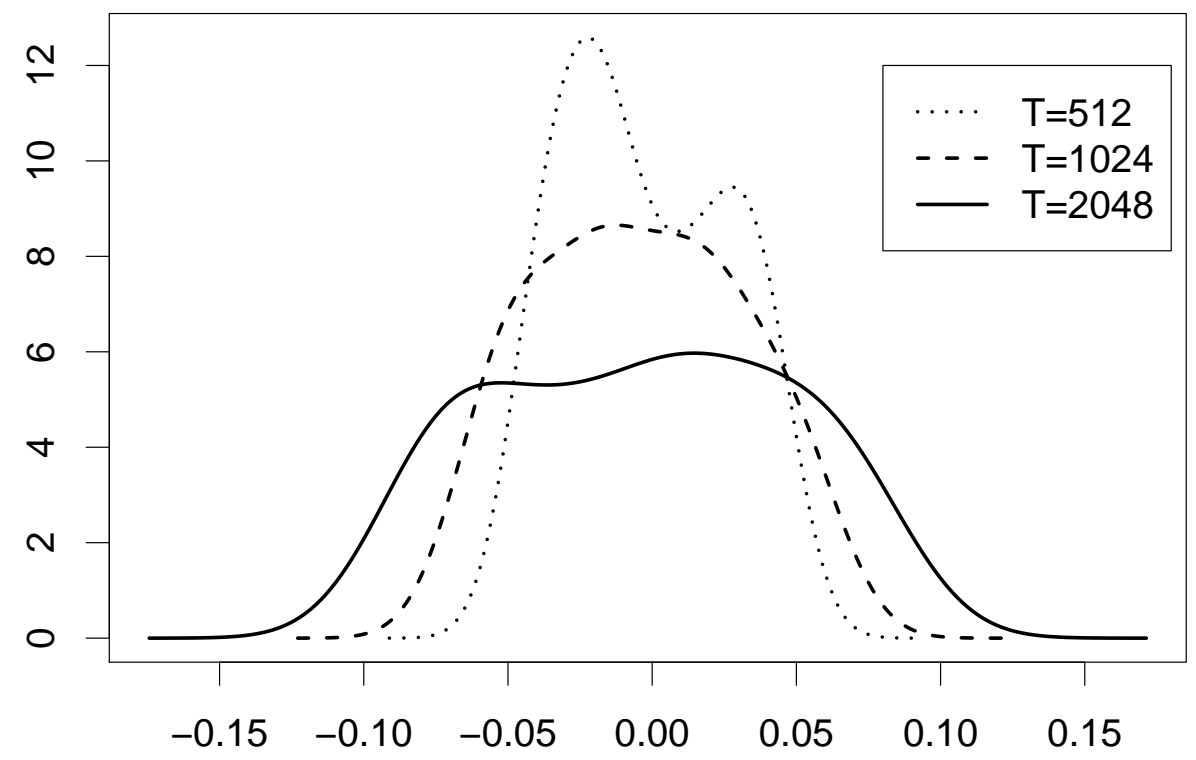

Figure 4: Density function estimates of $\sqrt{T}\left(\bar{\sigma}_{T}-\sigma_{0}\right)$ for $\theta_{0}=\left(\alpha_{0}, \beta_{0}, \sigma_{0}\right)=(0.1,0.1,0.1)$. 

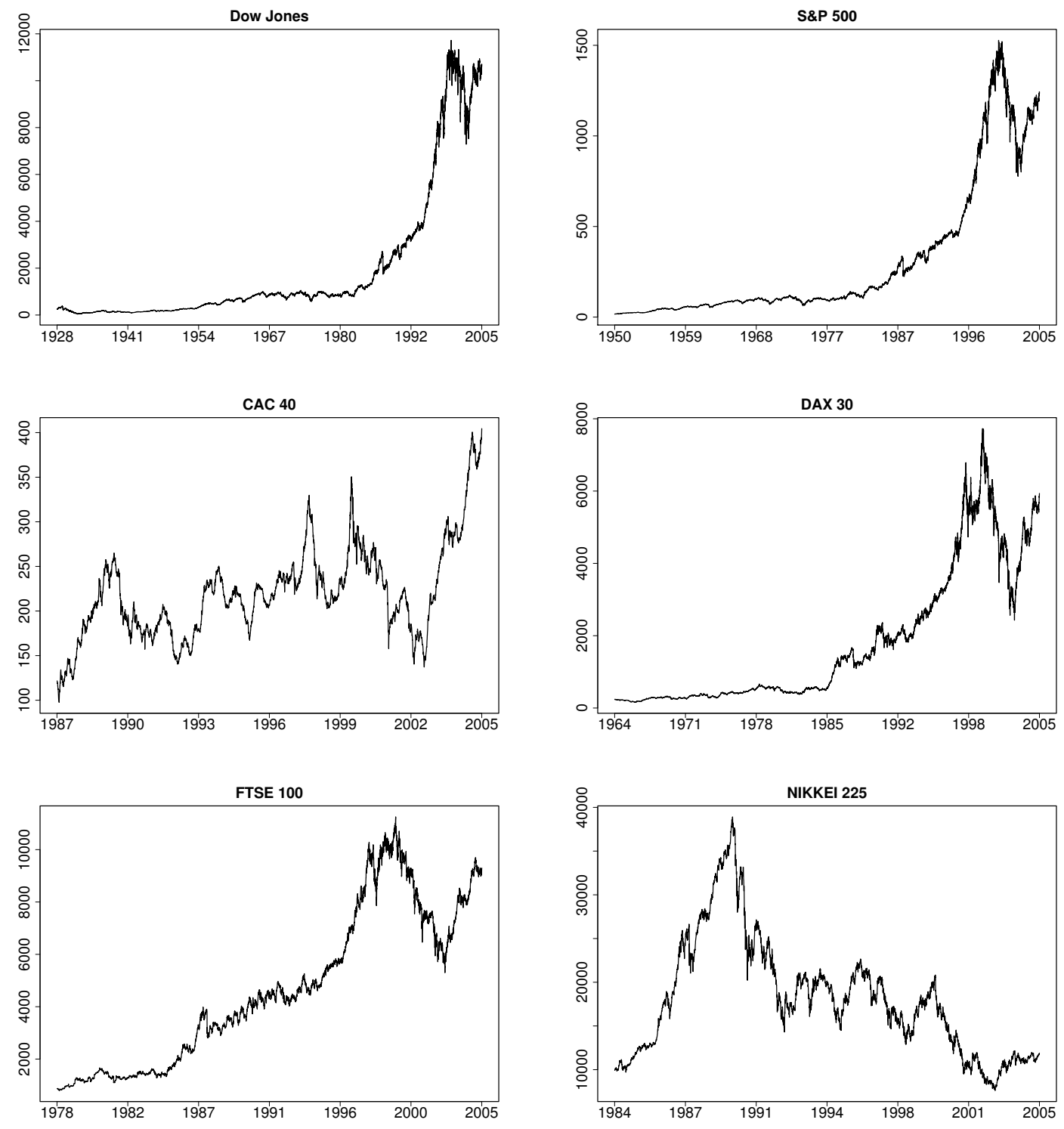

Figure 5: Financial Stock Indexes under study. 

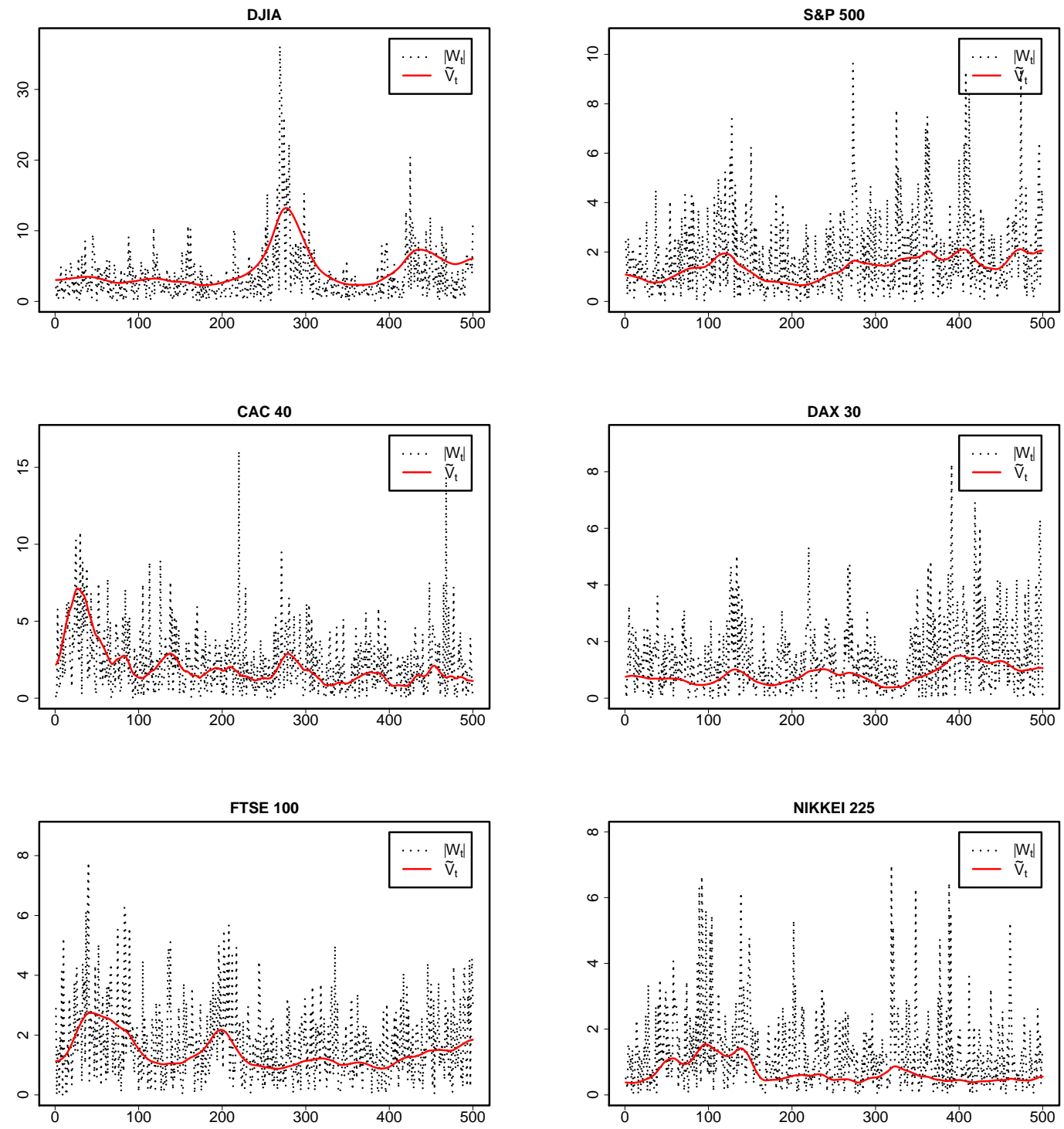

Figure 6: Estimation of the first 500 values of the volatility process of these stock market indexes. The dotted line represents the absolute value of the compounded returns divided by $\Delta=\frac{1}{250}$, i.e. $\left|W_{t}\right|$. 